This is a pre-copyedited, author-produced PDF of an article accepted for publication in Geophysical Journal Internation following peer review. The version of record is available:

Shane Zhang, Lili Feng, Michael H Ritzwoller, Three-station interferometry and tomography: coda versus direct waves, Geophysical Journal International, Volume 221, Issue 1, April 2020, Pages 521541, https://doi.org/10.1093/gjilggaa046. 


\title{
Three-Station Interferometry and Tomography: Coda vs. Direct Waves
}

\author{
Shane Zhang ${ }^{1}$, Lili Feng ${ }^{1}$, Michael H. Ritzwoller ${ }^{1}$ \\ ${ }^{1}$ Department of Physics, University of Colorado Boulder, Boulder, CO 80309, USA.
}

\begin{abstract}
Summary
Traditional two-station ambient noise interferometry estimates the Green's function between a pair of synchronously deployed seismic stations. Three-station interferometry considers records observed three stations at a time, where two of the stations are considered receiver-stations and the third is a source-station. Cross-correlations between records at the source-station with each of the receiver-stations are correlated or convolved again to estimate the Green's function between the receiver-stations, which may be deployed asynchronously. We use data from the EarthScope USArray in the western US to compare Rayleigh wave dispersion obtained from two-station and three-station interferometry. Three three-station interferometric methods are distinguished by the data segment utilized (coda-wave or direct-wave) and whether the source-stations are constrained to lie in stationary phase zones approximately inline with the receiver-stations. The primary finding is that the three-station direct wave methods perform considerably better than the three-station coda-wave method and two-station ambient noise interferometry for obtaining surface wave dispersion measurements in terms of signal-to-noise ratio, bandwidth, and the number of measurements obtained, but possess small biases relative to two-station interferometry. We present a ray-theoretic correction method that largely removes the bias below $40 \mathrm{~s}$ period and reduces it at longer periods. Three-station directwave interferometry provides substantial value for imaging the crust and uppermost mantle, and its ability to bridge asynchronously deployed stations may impact the design of seismic networks in the future.
\end{abstract}

Key words: Seismic noise; Seismic interferometry; Seismic tomography; Surface waves and free oscillations; Coda waves.

Corresponding author: Shane Zhang, shzh3924@colorado.edu 


\section{Introduction}

Inter-station seismic interferometry is designed to extract an estimate of the Green's function between pairs of seismic stations or receivers. Generally speaking, there are two established methods to perform this task, which we will call "two-station interferometry" and "three-station interferometry". In this paper, we attempt to discuss and characterize important variants of three-station interferometry, and compare the characteristics amongst the variants and to two-station interferometry using data from the EarthScope Transportable Array (TA) in the US.

Two-station interferometry is the traditional method of "ambient noise interferometry" or "ambient noise correlation". It is the more commonly applied method and is based on a single cross-correlation between ambient noise recorded at two stations. The cross-correlation can be converted to an estimate of the Green's function of the medium if the time series is long enough (e.g., Shapiro \& Campillo, 2004). In this case, one of the stations acts as a virtual source of the seismic energy and the other as the receiver. When many pairs of stations are considered, it is the basis for ambient noise tomography of surface waves, and many applications of this method have emerged since Shapiro et al. (2005); Sabra et al. (2005); Yao et al. (2006).

Three-station interferometry, in contrast, considers recordings from three seismic stations at a time. This method takes the cross-correlation between recordings of ambient noise at one station, which acts as a virtual source and which we call the "sourcestation", with recordings from two other stations, which are called the "receiver-stations". These two cross-correlations, or particular segments of them, are then cross-correlated again (or, as discussed further below, convolved). Stacking the resulting waveforms from many source-stations for the same pair of receiver-stations provides an estimate of the Green's function between the two receiver-stations. This method, therefore, is based on cross-correlations performed three at a time, where the last one has been referred to as the "correlation of correlations" (Stehly et al., 2008) but in certain circumstances will be a convolution of correlations. We refer to this method generally speaking as "threestation interferometry", to distinguish it from traditional two-station ambient noise methods. When the final cross-correlation is between the coda-wave parts of the first two correlations the method is commonly referred to as the "correlation of the coda of correlations" or $C^{3}$ (Stehly et al., 2008). 
Fig. 1 illustrates some of the notation introduced in this paper. For two-station interferometry, we denote the cross-correlation between a pair of seismograms observed at stations $r_{i}$ and $r_{j}$ as $C_{2}\left(r_{i}, r_{j}\right)$. With an appropriate phase-shift, $C_{2}\left(r_{i}, r_{j}\right)$ can be converted to an estimate of the Green's function between the two stations, $\hat{G}_{2}\left(r_{i}, r_{j}\right)$, where we suppress the time-dependence of the correlations and the estimated Green's function. For three-station interferometry, cross-correlations between observations at a source-station, $s_{k}(1 \leq k \leq N)$, with the two receiver-stations, $C_{2}\left(s_{k}, r_{i}\right)$ and $C_{2}\left(s_{k}, r_{j}\right)$, are correlated again (or in some circumstances convolved). This produces the three-station "sourcespecific interferogram", $C_{3}\left(r_{i}, r_{j} ; s_{k}\right)$, for source-station $s_{k}$. (The subscript "3" distinguishes the final cross-correlation or convolution from the first two correlations.) The "composite Green's function" for three-station interferometry is produced by taking a weighted sum over the contributing source-specific interferograms from the $N$ sourcestations:

$$
\hat{G}_{3}\left(r_{i}, r_{j}\right)=\sum_{k=1}^{N} w_{k} C_{3}\left(r_{i}, r_{j} ; s_{k}\right)
$$

where $w_{k}$ is a weight. $\hat{G}_{3}$ provides information about the medium between the two receiverstations. For this equation to hold, $C_{3}$ must have an appropriate phase-shift applied prior to the summation.

The advantages of two-station interferometry include its simplicity and general applicability. The principal advantage of the three-station method over the two-station method is that the two receiver-stations do not have to operate at the same time, although they do have to operate synchronously with each source-station for some length of time. Thus, three-station interferometry can be applied to asynchronously deployed stations (Ma \& Beroza, 2012; Curtis et al., 2012), which provides the opportunity for what Curtis et al. (2012) call "retrospective seismology". In terms of applications, the method will be most impactful in settings where there is a long-term backbone seismic network to provide the source-stations and shorter term deployments from which the receiver-stations are taken.

In practice, the data processing involves three noteworthy subtleties. (1) The crosscorrelations of seismic noise data that form the basis for both the two-station and threestation methods involve refined data processing methods that aim to speed convergence and reduce sensitivity to earthquakes and localized persistent noise sources (e.g., Ritzwoller \& Feng, 2019). We discuss the methods of data processing that we use in sections 
2 and 3 below, but we do not attempt to optimize data processing procedures for threestation interferometry.

(2) We must specify which parts of the cross-correlations of seismic noise, $C_{2}\left(s_{k}, r_{i}\right)$ and $C_{2}\left(s_{k}, r_{j}\right)$, that are correlated or convolved to produce the source-specific interferogram for source $s_{k}, C_{3}\left(r_{i}, r_{j} ; s_{k}\right)$. Fig. 2 identifies the two parts of the cross-correlations relevant to this study: the coda-wave $(\mathrm{CW})$ and the direct-wave (DW) parts. If coda waves are correlated, we refer to the method to produce an estimated Green's function as "codawave interferometry" and if direct waves are correlated or convolved we call it "directwave interferometry".

(3) Finally, it is important to specify how to determine the weights, $w_{k}$, that convert individual source-specific interferograms to the estimated Green's function. One aspect of the choice of weights is the geometrical relationship between the receiver-stations and each source-station. For coda-wave interferometry there is no geometrical constraint so that all source-stations are used for a given receiver-station pair irrespective of their relative position; that is, the geometrical-weights are all unity (Fig. 3a). However, for direct-wave interferometry we impose the constraint that the source-stations lie within appropriately defined "stationary phase zones" so that sources outside those zones are given zero geometrical-weight and sources inside the zones are given unit geometricalweight. The stationary phase zone is a Fresnel ellipse for source-stations between the receiverstations (Fig. 3c) or hyperbolae for source-stations not between the receiver-stations (Fig. 3b), where the receiver-stations are the foci of both the ellipse and the hyperbolae. Another aspect of these weights is based on a measure of the quality of each sourcespecific interferogram, $C_{3}\left(r_{i}, r_{j}, s_{k}\right)$. Both aspects of assigning weights are discussed in greater detail in section 3.2 .

It is useful to define nomenclature to distinguish the interferometric methods considered here. Traditional two-station ambient noise (AN) interferometry is denoted:

$$
\mathcal{I}_{2}^{A N}
$$

where the "2" represents the number of stations used. Three-station methods require the specification of two additional fields, "type" and "geometry", so that three-station interferometric methods are denoted generally as:

$$
\text { geometry } \mathcal{I}_{3}^{\text {type }}
$$


Here, "type" indicates either coda-wave (CW) or direct-wave (DW) interferometry, "geometry" represents the shape of the stationary phase zone, and the " 3 " indicates the number of stations used in the method prior to stacking over source-stations. Of course, in the stacking of eq. (1) multiple source-stations will typically be used, but data analysis is performed three stations at a time. There is no geometrical constraint for codawave interferometry; thus this field is left blank in this case. For direct-wave interferometry the geometrical constraint is either an ellipse (ell) or a hyperbola (hyp).

Therefore, we identify three general methods of three-station interferometry to estimate Green's functions. First, three-station coda-wave interferometry is denoted as

$$
\mathcal{I}_{3}^{C W}
$$

Hence, there is the following relationship between our notation and earlier notation: $\mathcal{I}_{3}^{C W} \equiv$ $C^{3}$. Second, three-station direct-wave interferometry with sources in the elliptical stationary phase zone between the receiver-stations is represented as

$$
{ }^{\text {ell }} \mathcal{I}_{3}^{D W}
$$

Finally, we indicate three-station direct-wave interferometry with sources in the hyperbolic stationary phase zones radially outside the receiver-stations as

$$
{ }^{\text {hyp }} \mathcal{I}_{3}^{D W}
$$

When we refer to direct-wave interferometry generally without distinguishing between the geometry of the stationary phase zones, we will use the symbol $\mathcal{I}_{3}^{D W}$, leaving the geometry field blank.

Three-station coda-wave interferometry $\left(\mathcal{I}_{3}^{C W}\right)$ was initiated by Stehly et al. (2008) and has been fairly well studied (Garnier \& Papanicolaou, 2009; Froment et al., 2011; Ma \& Beroza, 2012; Zhang \& Yang, 2013; Haendel et al., 2016; Sheng et al., 2017, 2018; Spica et al., 2017; Ansaripour et al., 2019). Applications of $\mathcal{I}_{3}^{C W}$ to surface wave tomography or 3-D model construction remain rare, however, in particular at regional or continental scales. To the best of our knowledge, the principal exceptions are two studies that combine group velocity measurements from $\mathcal{I}_{3}^{C W}$ with traditional ambient noise interferometry $\left(\mathcal{I}_{2}^{A N}\right)$ to improve 3-D models of Mexico and the southern US (Spica et al., 2016), and of the Iranian Plateau (Ansaripour et al., 2019).

In comparison, three-station direct-wave interferometry $\left(\mathcal{I}_{3}^{D W}\right)$ has received much less attention. Froment et al. (2011) discussed the possibility for using direct versus coda 
waves, and differentiated between two types of correlations of correlations: $C_{\text {coda }}^{3}$ and $C_{\text {all }}^{3}$, where $C_{\text {coda }}^{3}$ denotes the correlation of the coda of correlations and $C_{\text {all }}^{3}$ refers to correlating the entirety of the correlations. Thus, as noted above, their $C_{\text {coda }}^{3}$ is similar to our $\mathcal{I}_{3}^{C W}$ and because the direct-waves dominate the coda-waves in the correlations, their $C_{\text {all }}^{3}$ is in some ways similar to our $\mathcal{I}_{3}^{D W}$. They, however, do not discuss constraining the source-stations in direct-wave interferometry to lie in stationary phase zones, although other studies do (Curtis \& Halliday, 2010; Duguid et al., 2011; Curtis et al., 2012; Entwistle et al., 2015). Moreover, the latter studies also recognize that for the elliptical stationary phase zone, when source-stations lie generally between the receiver-stations, the original cross-correlations should be convolved with one another rather than crosscorrelated. Therefore, for ${ }^{h y p} \mathcal{I}_{3}^{D W}$ the three data operations are all cross-correlations, but for ${ }^{e l l} \mathcal{I}_{3}^{D W}$ the third data operation is a convolution. Discussion of the role of convolution in interferometry goes back at least to Slob and Wapenaar (2007). Entwistle et al. (2015) applied aspects of direct-wave interferometry to data from the EarthScope Transportable Array, but to the best of our knowledge $\mathcal{I}_{3}^{D W}$ has not yet been applied tomographically or in the context of inversions for 3-D models and its properties remain poorly understood.

The purpose of this paper is to determine and compare empirically the characteristics of the three-station methods to each other and to two-station interferometry. In particular, we focus on obtaining reliable surface wave dispersion measurements in the context of tomography. From the outset, it is evident that coda-wave interferometry has the advantage that any geometrical relationship can exist between the source-stations and the receiver-stations, whereas for direct-wave interferometry only a small subset of stations can be used as source-stations for each pair of receiver-stations. In coda-wave interferometry, however, signals emerge very slowly with the addition of source-stations, which means that many more source-stations are needed to recover reliable estimated Green's functions. Therefore, the relative merits of direct-wave interferometry and codawave interferometry (which of the methods will be preferable, in what ways, and in which settings) need to be determined empirically.

We address these questions by applying $\mathcal{I}_{2}^{A N}, \mathcal{I}_{3}^{C W},{ }^{\text {ell }} \mathcal{I}_{3}^{D W}$, and ${ }^{h y p} \mathcal{I}_{3}^{D W}$ across the central and western US to all stations west of $95^{\circ} \mathrm{W}$ longitude from the EarthScope Transportable Array to measure Rayleigh wave dispersion from $8 \mathrm{~s}$ to $80 \mathrm{~s}$ period and present associated phase speed maps from $10 \mathrm{~s}$ to $60 \mathrm{~s}$ period. We pay particular attention to 
the agreement between the three-station results and the two-station results, including systematic differences (bias) and fluctuation, and to the distributions of measurements as functions of signal-to-noise ratio (SNR), band-width, and the number of measurements produced for asynchronously deployed receiver-stations.

\section{Data}

Three-station interferometry $\left(\mathcal{I}_{3}\right)$ is based on data output from two-station interferometry $\left(\mathcal{I}_{2}\right)$. As the basis for the three-station interferometry in this study, we use the two-station database of ambient noise cross-correlations $\left(C_{2}\right)$ constructed by Shen and Ritzwoller (2016). Stations in the database of Shen and Ritzwoller (2016) extend across the contiguous US, but we use only a subset of them in the central and western US (west of $95^{\circ} \mathrm{W}$ longitude), which defines our region of study (Fig. 4). We use all 1047 EarthScope USArray stations in this region deployed from 2005 to 2010, including 979 Transportable Array (_US-TA) stations and 68 Reference Network (_US-REF) stations. We retain a two-station cross-correlation only if its signal-to-noise ratio (SNR) is greater than 10, where SNR is defined as the ratio of the maximum amplitude of the waveform in the time window of the direct fundamental Rayleigh wave to the root-mean square of the waveform in the coda-wave window (Fig. 2). SNR defined in this way is independent of frequency. Among the 547,581 possible combinations of pairs from the 1047 stations, $66 \%(364,103)$ operated synchronously so that two-station ambient noise interferometry could be employed. Of these, we retained 325,446 (89\%) cross-correlations that met the SNR criterion. In contrast, $34 \%(183,478)$ of the station-pairs were deployed asynchronously.

The deployment of the Transportable Array started from the West Coast and rolled eastward, with stations deployed temporarily for $\sim 2$ years (Fig. 4). This rolling pattern provides an ideal geometry for direct-wave interferometry with an elliptical stationary phase zone, ${ }^{e l l} \mathcal{I}_{3}^{D W}$, in which source-stations lie approximately between receiver-stations. In contrast, the Reference Network was deployed permanently and was scattered across the US with a station spacing of $\sim 300 \mathrm{~km}$. This is a good geometry for coda-wave interferometry, $\mathcal{I}_{3}^{C W}$, and direct-wave interferometry with a hyperbolic stationary phase zones, ${ }^{h y p} \mathcal{I}_{3}^{D W}$, in which source-stations lie approximately radially outward from receiverstations. 
Shen and Ritzwoller (2016) used a common method of ambient noise data processing (Bensen et al., 2007). Briefly, continuous records of vertical component seismograms are cut to day-long segments and downsampled from $40 \mathrm{~Hz}$ to $1 \mathrm{~Hz}$. Then the instrument response, mean and trend are removed. To minimize the effects of strong directional sources (in particular earthquakes) and to broaden the usable bandwidth, temporal normalization and spectral whitening are applied. The temporal normalization uses a $80 \mathrm{~s}$ running time window, which strongly attenuates signals with periods above $80 \mathrm{~s}$. For this reason we will focus our interpretation on measurements only up to $80 \mathrm{~s}$ period and show tomographic results only up to $60 \mathrm{~s}$ period.

After pre-processing, daily seismograms from all available combinations of stationpairs $\left(r_{i}, r_{j}\right)$ are cross-correlated to produce $C_{2}\left(r_{i}, r_{j}\right)$, between correlation lag times of $\pm 3000 \mathrm{~s}$. Daily correlations are then stacked to generate two-station estimated Green's functions between each pair of stations $\left(\hat{G}_{2}\left(r_{i}, r_{j}\right)\right)$. Finally, we compute the so-called "symmetric component" of the estimated Green's function by averaging the estimated Green's function at positive and negative correlation lags for simplicity. We will also refer to this symmetric component estimated Green's function as $\hat{G}_{2}\left(r_{i}, r_{j}\right)$, even though it is defined only for positive lag. This database of symmetric component estimated Green's functions is the basis for the three-station analysis (section 3).

\section{Data Processing for Three-Station Interferometry}

The input for three-station interferometry are the two-station symmetric component cross-correlations (or estimated Green's functions) taken from the database of Shen and Ritzwoller (2016) with SNR > 10. As inter-station cross-correlations, these functions are denoted by $C_{2}$ and as estimated Green's functions by $\hat{G}_{2}$. Three-station sourcespecific interferograms $\left(C_{3}\right)$ are cross-correlations of the coda-wave parts of the interstation cross-correlations, or cross-correlations or convolutions of the direct-wave parts of the inter-station cross-correlations. Three-station data processing aims to compute the composite Green's function between pairs of receiver-stations by stacking the threestation interferograms over contributions from various source-stations.

For concreteness, consider a receiver-station pair $\left(r_{i}, r_{j}\right)$ and a set of source-stations, $\left\{s_{k}\right\}_{k=1}^{N}$, that operate synchronously with both $r_{i}$ and $r_{j}$ at least for some time. Fig. 1b depicts this situation, where one source-station is shown. Let the coda-wave parts 
of the two-station cross-correlations be denoted $C_{2}^{C W}\left(s_{k}, r_{i}\right)$ and $C_{2}^{C W}\left(s_{k}, r_{j}\right)$, and the direct-wave parts be written $C_{2}^{D W}\left(s_{k}, r_{i}\right)$ and $C_{2}^{D W}\left(s_{k}, r_{j}\right)$, where the coda-wave and direct-wave segments are defined in Fig. 2. The three-station data processing procedure breaks into three principal steps (sections 3.1 - 3.3).

\subsection{Constructing Source-Specific Interferograms}

The first step in three-station data processing is devoted to cross-correlating or convolving segments of the two-station cross-correlations. It is broken into three categories depending on whether one considers the direct- or coda-wave segments of the two-station cross-correlations and the geometrical relationship between the receiver-station pair and each source-station. For direct-waves, the geometrical relationship is summarized in terms of hyperbolic or elliptical stationary phase zones (Fig. 3b,c).

(1) The first category is, for each source-station, to compute the three-station sourcespecific interferograms based on the coda-waves in the two-station cross-correlations. That is, correlate $C_{2}^{C W}\left(s_{k}, r_{i}\right)$ and $C_{2}^{C W}\left(s_{k}, r_{j}\right)$ for all $s_{k}$ to produce $C_{3}^{C W}\left(r_{i}, r_{j} ; s_{k}\right)$ for $1 \leq$ $k \leq N$. An example record-section containing three-station coda-wave source-specific interferograms is presented in Fig. 5a, where each trace is for a separate source-station.

(2) The second category is to compute the three-station source-specific interferograms based on the direct-waves in the two-station cross-correlations for the source-stations in the hyperbolic stationary phase zones. For each source-station $s_{k}$ in the stationaryphase hyperbolae for the receiver-station-pair, cross-correlate $C_{2}^{D W}\left(s_{k}, r_{i}\right)$ and $C_{2}^{D W}\left(s_{k}, r_{j}\right)$ to produce ${ }^{h y p} C_{3}^{D W}\left(r_{i}, r_{j} ; s_{k}\right)$. An example record-section for three-station direct-wave source-specific interferograms computed by cross-correlation is shown in Fig. 5b, where each trace is for a separate source-station. For this record-section, cross-correlations are computed based on source-stations irrespective of whether they lie in the stationary-phase hyperbolae. However, the green-shaded regions identify the stationary phase zones.

(3) The third category is similar to the second, but we compute the three-station source-specific interferograms based on the direct-waves in the two-station cross-correlations for the source-stations in the elliptical stationary phase zone. For each source-station $s_{k}$ in the stationary-phase ellipse for this receiver-station-pair, convolve $C_{2}^{D W}\left(s_{k}, r_{i}\right)$ and $C_{2}^{D W}\left(s_{k}, r_{j}\right)$ to produce ${ }^{\text {ell }} C_{3}^{D W}\left(r_{i}, r_{j} ; s_{k}\right)$. An example record-section for three-station source-specific direct-wave interferograms computed by convolution is shown in Fig. 5c, 
where each trace is for a separate source-station. As in Fig. 5b, convolutions are presented irrespective of whether the source-station lies in the stationary-phase ellipse, but the green-shaded region identifies the stationary phase zone.

Convolution of the direct-wave parts of the two-station records when source-stations lie in the elliptical stationary phase zone has been formally justified by other studies (Halliday \& Curtis, 2009; Curtis \& Halliday, 2010). We provide a heuristic argument for illumination. When a source-station lies radially outward from a pair of receiver-stations, it is the time-difference between the travel times from the source-station to the two receiverstations that approximates the travel time between the two receiver-stations. Cross-correlation of two records finds the time-difference between them, therefore when source-stations lie outside the receiver-stations it is the appropriate method to apply. In contrast, convolutions find the sum of the times. When a source-station lies between two receiver-stations, we wish to find the sum of the times from the source-station to each receiver-station, so that convolution is the appropriate method to apply in this case.

We define the hyperbolic and elliptical stationary phase zones in a straightforward and simplified manner. An ellipse is defined as the locus of points where the sum of the distances to the foci is constant. Let $d_{i j}$ be the great-circle distance between the two receiverstations, $d_{k i}$ be the distance between a point $s_{k}$ on the ellipse and receiver-station $r_{i}$, and $d_{k j}$ be the distance between $s_{k}$ and receiver-station $r_{j}$. Then we define the elliptical stationary phase zone for method ${ }^{e l l} \mathcal{I}_{3}^{D W}$ as

$$
d_{k i}+d_{k j} \leq(1+\alpha) d_{i j},
$$

where $\alpha \geq 0$ and we choose $\alpha=10^{-2}$. Thus, if source-station $s_{k}$ lies within the elliptical stationary phase zone, the sum of distances from $s_{k}$ to $r_{i}$ and to $r_{j}$ is less than $1 \%$ longer than the distance between the receiver-stations.

Similarly, a hyperbola is defined as the locus of points where the difference of the distances to the foci is constant. We therefore define the hyperbolic stationary phase zones for method ${ }^{h y p} \mathcal{I}_{3}^{D W}$ as

$$
\left|d_{k i}-d_{k j}\right| \geq(1-\alpha) d_{i j}
$$

where $\alpha \in[0,1]$ and again we choose $\alpha=10^{-2}$. This means that if source-station $s_{k}$ lies within the hyperbolic stationary phase zone, the difference of distances from $s_{k}$ to $r_{i}$ and to $r_{j}$ is greater than $99 \%$ of the distance between the receiver-stations. On a sphere, 
the locus of points where the difference of the distances to the foci is constant, however, approximates a hyperbola only near the foci.

The stationary phase zones can be defined alternatively using azimuthal angle $\theta$ (Fig. 3) instead of $\alpha$. For the methods $\mathcal{I}_{3}^{C W}$ and ${ }^{h y p} \mathcal{I}_{3}^{D W}, \theta$ is the angle from the sourcestation to the mid-point between the receiver-stations (Fig. 3a,b), which defines the slopes of the asymptotes of a hyperbola. It is related to $\alpha$ by $\cos \theta=1-\alpha$, where $\theta \in[0,2 \pi]$. The definition of angle $\theta$ for a given source-station for method ${ }^{e l l} \mathcal{I}_{3}^{D W}$ is motivated by the symmetry in eqs. (4) and (5) below. To do so, first identify the ellipse on which the source-station lies with the two receiver-stations as foci. Then find the intersection point between the ellipse and the perpendicular bisector of the line segment linking the two receiver-stations. Angle $\theta$ is the angle between a receiver-station and this intersection point. Fig. 3c shows an example of this intersection point, but does not identify the location of the source-station or the ellipse on which it lies. In this case, $\theta$ is related to $\alpha$ by $\cos \theta=1 /(1+\alpha)$, where $\theta \in\left[0, \frac{\pi}{2}\right]$. For the same $\alpha, \theta$ is generally larger for ${ }^{h y p} \mathcal{I}_{3}^{D W}$ than for ${ }^{\text {ell }} \mathcal{I}_{3}^{D W}$. Our choice of $\alpha=10^{-2}$ corresponds to a maximum $\theta \approx 8^{\circ}$ for both ${ }^{h y p} \mathcal{I}_{3}^{D W}$ and ${ }^{\text {ell }} \mathcal{I}_{3}^{D W}$

We use eqs. (2) and (3) with $\alpha=10^{-2}$ to define the stationary phase zones in this paper for methods ${ }^{e l l} \mathcal{I}_{3}^{D W}$ and ${ }^{h y p} \mathcal{I}_{3}^{D W}$, respectively. These definitions are chosen for simplicity and because they appear to provide reliable results in the applications we consider. However, the choice of the value of $\alpha$ is ad-hoc as is its frequency-independence. More elaborate, perhaps frequency-dependent, definitions may prove to be preferable. The approximate arrival time, $\delta t$, for method ${ }^{h y p} \mathcal{I}_{3}^{D W}$ is known (Tsai, 2009; Yao \& van der Hilst, 2009):

$$
\delta t=\frac{d_{i j}}{v} \cos \theta
$$

for a plane-wave in a medium with constant wave speed $v$, where $d_{i j}$ is the inter-receiverstation distance and $\theta$ is shown in Fig. 3b. The grey line plotted in Fig. $\mathbf{5 b}$ is for this formula. Analogously, the approximate arrival time $t_{\text {sum }}$ for method ${ }^{e l l} \mathcal{I}_{3}^{D W}$ is:

$$
t_{\text {sum }}=\frac{d_{i j}}{v} \sec \theta
$$

for $\theta$ shown in Fig. 3c. The grey line plotted in Fig. 5c is for this formula. 


\subsection{Stacking Weights}

Appropriate stacking weights $w_{k}$ must be applied for each source-station $s_{k}$ to compute the composite Green's functions for each of the three-station methods $\left(\mathcal{I}_{3}^{C W},{ }^{\text {hyp }} \mathcal{I}_{3}^{D W}\right.$ and $\left.{ }^{e l l} \mathcal{I}_{3}^{D W}\right)$. The principal weight that we use is to set $w_{k}$ equal to the reciprocal of the rootmean-square (rms) of the noise in the coda-wave part of each source-specific interferogram, $C_{3}\left(r_{i}, r_{j} ; s_{k}\right)$ for receiver-stations $r_{i}$ and $r_{j}$. Defined in this way, we down-weight each contributing cross-correlogram by the rms of trailing noise. We do not, however, normalize the amplitude of the cross-correlograms. Therefore, down-weighting by the rms of trailing noise is approximately equivalent to normalizing the amplitudes of the crosscorrelograms then weighting by peak signal-to-rms trailing noise ratio (SNR). Because the peak signal grows approximately linearly with the time series length of the records used to compute the cross-correlations, and rms trailing noise grows approximately as the square root of the time series length, SNR grows approximately as the square root of time series length (Snieder, 2004; Bensen et al., 2007). Thus, the use of this weighting scheme tends to accentuate the contribution from longer cross-correlations, but less strongly than if we had not normalized by peak amplitude and inversely by the rms of the trailing noise.

There are three other aspects of the data processing that can be considered to be stacking weights. First, for the direct-wave three-station methods, we only include a sourcestation in the stack if it lies within an appropriately defined stationary phase zone, which is referred to as geometrical-weighting in the Introduction. This choice can be thought of as applying binary weights to source-stations depending on their position relative to the receiver-stations. Second, also as mentioned above, unless the two constituent twostation interferograms, $C_{2}\left(s_{k}, r_{i}\right)$ and $C_{2}\left(s_{k}, r_{j}\right)$, both have SNR $\geq 10$, the weight of the corresponding three-station interferogram, $C_{3}\left(r_{i}, r_{j} ; s_{k}\right)$, is set to zero; otherwise it is unity. Third, to include signals for the longest paths $(>3000 \mathrm{~km})$ in the coda-wave three-station method, a source-station is excluded if the length of either $C_{2}^{C W}\left(s_{k}, r_{i}\right)$ or $C_{2}^{C W}\left(s_{k}, r_{j}\right)$ is less than $1500 \mathrm{~s}$.

\subsection{Estimating Composite Green's Functions}

To compute the composite Green's function, $\hat{G}\left(r_{i}, r_{j}\right)$, for each of the three-station methods we apply the weighted sum given by eq. (1) based on the stacking weights (sec- 
tion 3.2). Fig. 6 provides some examples using the same pair of receiver-stations used in the record-sections of Fig. 5 .

Fig. 6a presents an example composite Green's function for three-station codawave interferometry $\left(\mathcal{I}_{3}^{C W}\right)$. For this method, no stationary phase zone is needed, so contributions from all source-stations are included in the stack. This is the black line in Fig. 6a, labelled "Stack all", which is compared to the two-station ambient noise cross-correlation plotted as the red line and labelled $\mathcal{I}_{2}^{A N}$. Two observations of noteworthy: First, one of the features of coda-wave interferometry is the tendency for the composite Green's functions to be more symmetric than for two-station ambient noise methods (e.g., Stehly et al., 2008, and many others), and this is also observed in this example. We found it, however, to be an artifact due to the use of symmetric components (Sheng et al., 2018). Second, the SNR of the three-station coda-wave composite Green's function is lower than for the two-station record, even though in this case 510 source-stations contribute to the three-station interferogram. This highlights another aspect of coda-wave interferometry, i.e., signals emerge from noise very slowly as source-stations are introduced. And, as can be seen in Fig. 5a, constituent source-specific three-station interferograms are typically very noisy so that signals cannot be discerned in any of them. This implies that the presence of many long duration source-stations may be necessary for coda-wave interferometry to play a useful role in ambient noise interferometry, unless more sophisticated data processing procedures are applied (section 7.3). For comparison, we also plot in Fig. 6a the recovered composite Green's function based on source-stations that lie exclusively in the hyperbolic stationary phase zone. The choice of source-stations in this zone further degrades the SNR of the composite Green's function, indicating that there is no geometrical advantage to choosing source-stations in the end-fire directions in coda-wave interferometry.

Fig. 6 b shows an example composite Green's function for three-station direct-wave interferometry where the source-stations lie in the hyperbolic stationary phase zone $\left({ }^{h y p} \mathcal{I}_{3}^{D W}\right)$. In this case, the green line, which is the stack for source-stations only in the hyperbolic stationary phase zones, is the Green's function estimate, and there are 25 source-stations. Retaining source-stations at all azimuths (black line) degrades the result by adding precursory noise. Two comments are worthy of note in comparing the three-station composite Green's function (green line) with two-station Green's function (red line). First, the relative amplitudes for the different correlation lags are more similar than for coda- 
waves. Second, precursory noise is lower for the three-station estimate. These are both common characteristics when comparing two-station to three-station Green's functions.

Finally, Fig. 6c presents an example composite Green's function for three-station direct-wave interferometry where the source-stations lie in the elliptical stationary phase zone $\left({ }^{e l l} \mathcal{I}_{3}^{D W}\right)$. The green line, which is the stack for source-stations only in the elliptical stationary phase zones, is the composite Green's function estimate, and there are 7 source-stations. As with the hyperbolic stationary phase zone, retaining source-stations at all azimuths (black line) degrades the result but in this case adds both precursory and trailing noise, especially the trailing noise. In this case, too, there is lower precursory noise for the three-station estimate than for $\mathcal{I}_{2}^{A N}$.

\section{Dispersion Measurements}

\subsection{Frequency-Time Analysis}

To measure frequency dependent phase speed, we apply frequency-time analysis (FTAN; Dziewonski et al., 1969; Levshin \& Ritzwoller, 2001; Bensen et al., 2007). We assume that the measured phase of a two-station interferogram $\left(\mathcal{I}_{2}^{A N}\right)$ at frequency $\omega$ in the frequency domain for receiver-stations $r_{i}$ and $r_{j}$ is approximately (Lin et al., 2008)

$$
\phi_{i j}^{A N}(\omega)=\frac{\omega}{c_{i j}} d_{i j}+\frac{\pi}{4}+\phi_{s}+2 N \pi, N \in \mathbb{Z}
$$

where $d_{i j}$ is the distance between the two receiver-stations, $\pi / 4$ is from the far-field or high-frequency asymptotic approximation of the Bessel function, $\phi_{s}$ is an initial phase term, and $c_{i j}$ is the frequency-dependent phase speed, which is what we aim to measure.

For two-station ambient noise interferometry $\left(\mathcal{I}_{2}\right), \phi_{s} \approx 0$ has been shown theoretically (Snieder, 2004) and empirically (Yao et al., 2006; Lin et al., 2008). For threestation coda-wave interferometry $\left(\mathcal{I}_{3}^{C W}\right), \phi_{s}$ should also be approximately 0 . However, for three-station direct-wave interferometry $\left(\mathcal{I}_{3}^{D W}\right), \phi_{s}$ will differ from 0 , and this initial phase must be taken into account when measuring phase speed.

For ${ }^{h y p} \mathcal{I}_{3}^{D W}$, let the source-station $s_{k}$ lie outside the two receiver-stations at distances $d_{k i}$ from $r_{i}$ and $d_{k j}$ from $r_{j}(\mathbf{F i g}$. $7 \mathbf{b})$. Because correlation of two interferograms will determine the difference of the phases in the frequency domain, the phase of ${ }^{h y p} C_{3}\left(r_{i}, r_{j} ; s_{k}\right)$ 
is

$$
{ }^{h y p} \phi_{i j ; k}=\phi_{k i}^{A N}-\phi_{k j}^{A N}=\omega\left(\frac{d_{k i}}{c_{k i}}-\frac{d_{k j}}{c_{k j}}\right)+2 N \pi .
$$

From straight-ray and far-field assumptions (Tsai, 2009), we have

$$
\frac{d_{k i}}{c_{k i}}-\frac{d_{k j}}{c_{k j}} \approx \frac{d_{k i}-d_{k j}}{c_{i j}}
$$

thus

$$
{ }^{h y p} \phi_{i j ; k}=\frac{\omega}{c_{i j}}\left(d_{i j}+{ }^{h y p} \delta d_{i j ; k}\right)+2 N \pi
$$

where

$$
{ }^{h y p} \delta d_{i j ; k}=d_{k i}-d_{k j}-d_{i j}
$$

For ${ }^{\text {ell }} \mathcal{I}_{3}^{D W}$, source-stations lie generally between the two receiver-stations (Fig.

7a). Because convolution of two interferograms will determine the sum of the phases in the frequency domain, the phase of ${ }^{e l l} C_{3}\left(r_{i}, r_{j} ; s_{k}\right)$ is

$$
{ }^{e l l} \phi_{i j ; k}=\phi_{k i}^{A N}+\phi_{k j}^{A N}=\omega\left(\frac{d_{k i}}{c_{k i}}+\frac{d_{k j}}{c_{k j}}\right)+\frac{\pi}{2}+2 N \pi .
$$

Based on approximations similar to ${ }^{h y p} \mathcal{I}_{3}^{D W}$, we find

$$
{ }^{e l l} \phi_{i j ; k}=\frac{\omega}{c_{i j}}\left(d_{i j}+{ }^{e l l} \delta d_{i j ; k}\right)+\frac{\pi}{2}+2 N \pi
$$

where

$$
{ }^{e l l} \delta d_{i j ; k}=d_{k i}+d_{k j}-d_{i j}
$$

Assuming ${ }^{h y p} \delta d=0$ gives ${ }^{h y p} \phi_{s}=-\pi / 4$ by comparing eqs. (6) and (9). Similarly, assuming ${ }^{e l l} \delta d=0$ yields ${ }^{e l l} \phi_{s}=\pi / 4$ by comparing eqs. (6) and (12). The assumption that $\delta d=0$ will lead to biased measurements for the three-station direct-wave methods and its correction is discussed in section 5.

Fig. 8 compares example frequency-time (FTAN) diagrams for the two-station method and the three-station methods, for the two receiver-stations M07A and M15A. The four diagrams are similar at short periods but the diagrams for the two direct-wave methods show larger relative amplitudes at longer periods. For the coda-wave diagram, longer periods are too noisy to measure and the $26 \mathrm{~s}$ stripe correspond to a spatially localized microseism source. The effects of the $26 \mathrm{~s}$ microseism are discussed in section 7.2.

We apply two additional quality control criteria to the dispersion measurements. First, for a dispersion measurement to be retained, we apply a spectral SNR (Bensen et 
al., 2007) criterion to the composite Green's function, where again SNR is defined as the peak amplitude in the direct-wave window divided by the rms of the waveform in the coda-wave window. That is, at a given period the composite Green's function must have a SNR $\geq 10$ otherwise the dispersion measurement at that period is discarded. Second, the distance between the two receiver-stations must be greater than three wavelengths (Lin et al., 2008) for the dispersion measurement to be retained. For example, if the phase speed is $4 \mathrm{~km} / \mathrm{s}$, at $20 \mathrm{~s}$ period the receiver-stations must be separated by more than 240 $\mathrm{km}$. This criterion becomes more restrictive as period increases.

\subsection{General Characteristics}

Fig. 9a summarizes the spectral signal-to-noise ratio (SNR) of each of the four interferometric methods, averaging over the entire data set of dispersion measurements. Generally speaking, SNR decreases with period and the trends are similar between $\mathcal{I}_{3}$ and $\mathcal{I}_{2}^{A N}$. The peaks near $16 \mathrm{~s}$ and $8 \mathrm{~s}$ periods correspond to the primary and secondary microseisms, respectively, while the dip near 26 s period corresponds to the existence of a spatially localized microseismic source (e.g., Shapiro et al., 2006; Xia et al., 2013). Fig. 9b presents the SNR results relative to the SNR for $\mathcal{I}_{2}^{A N}$. The SNR for ${ }^{\text {ell }} \mathcal{I}_{3}^{D W}$ is slightly larger than for ${ }^{{ }^{h y p}} \mathcal{I}_{3}^{D W}$, while both have a SNR more than twice that of $\mathcal{I}_{2}^{A N}$ across a broad bandwidth. In contrast, $\mathcal{I}_{3}^{C W}$ has a much lower median SNR $(<10)$ across all periods.

Because SNR plays a significant role in the quality control of dispersion measurements, the number of accepted $\mathcal{I}_{2}$ and $\mathcal{I}_{3}$ measurements varies with period similar to SNR (Fig. 10a). The number of accepted $\mathcal{I}_{3}$ measurements can be divided into three categories depending on whether the two receiver-stations operated at the same time (synchronously) and whether an $\mathcal{I}_{2}$ measurement exists for the path so that the $\mathcal{I}_{3}$ measurement is new or repeated. These three categories of $\mathcal{I}_{3}$ measurements are referred to as "Synchronous-Repeated" (receiver-stations deployed synchronously, with both an $\mathcal{I}_{3}$ and an $\mathcal{I}_{2}$ measurement), "Synchronous-New" (receiver-stations deployed synchronously, with an $\mathcal{I}_{3}$ but not an $\mathcal{I}_{2}$ measurement), and "Asynchronous-New" (receiver-stations deployed asynchronously, with only an $\mathcal{I}_{3}$ measurement). In the Synchronous-New case, the receiverstations produced an $\mathcal{I}_{2}$ measurement but it was rejected, usually because it did not meet the SNR requirement. The numbers of $\mathcal{I}_{3}$ measurements that derive from these three categories are shown in Fig. 10b-d. In all categories, ${ }^{\text {ell }} \mathcal{I}_{3}^{D W}$ measurements somewhat 
outnumber the ${ }^{h y p} \mathcal{I}_{3}^{D W}$ measurements, and both outnumber the $\mathcal{I}_{2}^{A N}$ measurements (in cases where they exist) and greatly outnumber the $\mathcal{I}_{3}^{C W}$ measurements.

Fig. 10b is for the Synchronous-Repeated category of $\mathcal{I}_{3}$ measurements. By definition, the number of $\mathcal{I}_{3}$ measurements will be no larger than the number of $\mathcal{I}_{2}$ measurements. Nearly every existing $\mathcal{I}_{2}^{A N}$ measurement is accompanied by an $\mathcal{I}_{3}^{D W}$ measurement, but the number of $\mathcal{I}_{3}^{C W}$ measurements is considerably smaller. The number of these measurements generally decreases with period after maximizing between 20 and $30 \mathrm{~s}$, although the $\mathcal{I}_{3}^{C W}$ measurement maximizes nearer to $15 \mathrm{~s}$ period and decays very rapidly at longer periods.

Fig. 10c is for the Synchronous-New category of $\mathcal{I}_{3}$ measurements, and illustrates that many new longer periods measurements emerge from the $\mathcal{I}_{3}^{D W}$ method. Above about $50 \mathrm{~s}$ period, $\mathcal{I}_{3}^{D W}$ nearly doubles the number of measurements between synchronously deployed stations. Although a principal attraction of the three-station methods is the ability to obtain measurements between asynchronously deployed stations, but many new measurements result from the $\mathcal{I}_{3}^{D W}$ methods even for synchronously deployed stations particularly at long periods. There are essentially no new measurements from $\mathcal{I}_{3}^{C W}$ in this category.

Fig. 10d is for the Asynchronous-New category of $\mathcal{I}_{3}$ measurements, measurements from the $\mathcal{I}_{3}$ methods that are inherently non-existent for $\mathcal{I}_{2}$. Relative to the number of measurements delivered by $\mathcal{I}_{2}^{A N}$, the greatest impact of the $\mathcal{I}_{3}$ methods is at the longer periods of the bandwidth considered. The vast majority of the measurements for $\mathcal{I}_{3}^{C W}$ are from synchronously deployed stations (Fig. 10b), indicating that it is difficult for $\mathcal{I}_{3}^{C W}$ to bridge asynchronous stations.

\section{Correcting the Bias in Three-Station Direct-Wave Interferometry $\left(\mathcal{I}_{3}^{D W}\right)$}

As described above, the three-station methods are based on measuring the phase speed of the composite Green's function (eq. (1)), $\hat{G}_{3}\left(r_{i}, r_{j}\right)$, between a pair of receiverstations $\left(r_{i}, r_{j}\right)$, which is a stack of source-specific interferograms, $C_{3}\left(r_{i}, r_{j} ; s_{k}\right)$, that emerge from particular source-stations $s_{k}$. In direct-wave methods ${ }^{h y p} \mathcal{I}_{3}^{D W}$ and ${ }^{e l l} \mathcal{I}_{3}^{D W}$, the phase speed, $c_{i j}$, is measured using the composite Green's function based on eqs. (9) and (12), respectively, under the assumption that $\delta d=0$. It is this assumption for the composite Green's function that can produce the systematic bias in the three-station direct-wave 
methods. Because ${ }^{e l l} \delta d$ is always positive, assuming ${ }^{e l l} \delta d=0$ will result in a phase speed that is biased slow for ${ }^{e l l} \mathcal{I}_{3}^{D W}$. In contrast, because ${ }^{h y p} \delta d$ is always negative, assuming ${ }^{h y p} \delta d=0$ will result in a phase speed that is biased fast for ${ }^{h y p} \mathcal{I}_{3}^{D W}$.

Therefore, the correct distance to be used in measuring phase speed will depend on the specific location of each source-station. The direct use of the composite Green's function invariably will yield a biased phase speed measurement. To "de-bias" the phase speed measurements, we abandon the composite Green's function and measure a phase speed curve for each source-specific interferogram $\left(C_{3}\left(r_{i}, r_{j} ; s_{k}\right)\right)$ independently based on corrections from the more accurate ray-theoretic distance, ${ }^{e l l} \delta d_{i j ; k}$ or ${ }^{h y p} \delta d_{i j ; k}$, and then average the resulting phase speed curves.

Fig. 11 presents an example of the set of source-specific phase speed curves that have been de-biased by using the source-specific ray-theoretic distances. At each period we reject a source-specific measurement if its SNR $<10$ or either of the source-receiver distances is $<2 \lambda$. We do not, however, apply the wavelength criterion to the two sourcereceiver distances in constructing the composite Green's function before the de-biasing correction (section 3.3) because that would require stacking over different source-stations at different periods. Then we reject the $10 \%$ of measurements most different from the mean. Finally, we calculate the standard deviation $(\sigma)$ and discard the mean measurement altogether if $\sigma>60 \mathrm{~m} / \mathrm{s}$.

Fig. 12a shows the correction averaged over the entire data set for the two threestation direct-wave methods. Our definition of stationary phase zones that $\alpha=1 \%$ (eqs. (2) and (3)) provides an upper limit on the bias as $1 \%$. The absolute mean correction is about $10 \mathrm{~m} / \mathrm{s}$ at all periods for both methods, which is around $0.3 \%$, and thus consistent with the definition of stationary phase zones. The average standard deviation amongst the constituent source-specific curves over the entire data set is presented in Fig. 12b. The standard deviations for the ${ }^{\text {ell }} \mathcal{I}_{3}^{D W}$ method are generally smaller for the ${ }^{\text {hyp }} \mathcal{I}_{3}^{D W}$ method, consistent with the latter having larger and more complex sensitivity zones (section 7.1). These standard deviations may serve in the future as uncertainty estimates for the resulting dispersion measurements. 


\section{Validate Three-Station $\left(\mathcal{I}_{3}\right)$ against Two-Station $\left(\mathcal{I}_{2}\right)$ Interferometry}

To test if three-station methods are consistent with two-station interferometry, and if the de-biasing correction for $\mathcal{I}_{3}^{D W}$ presented in section $\mathbf{5}$ is effective, we statistically compare the differences in Rayleigh wave phase speed measurements and also the associated phase speed maps from the methods.

\subsection{Phase Speed Measurements}

Fig. 13 and Table 1 present comparisons of Rayleigh wave phase speed measurements derived from the three-station methods to two-station interferometry for common receiver-station pairs.

Fig. 13c and Table 1 (column 2) show that the mean difference between the twostation Green's functions and the three-station composite Green's functions based on codawaves is negligible $(<2 \mathrm{~m} / \mathrm{s}$, on average), from which we infer that the three-station method based on coda-waves is unbiased. The standard deviation of the difference decreases with period to achieve a minimum around $15 \mathrm{~s}$, but then increases rapidly with period although results extend only up to 30 s period.

In contrast, Fig. $\mathbf{1 3 a}, \mathbf{b}$ and Table $\mathbf{1}$ (columns $4 \& 6$ ) show the existence of a nonzero systematic difference or bias between each of the three-station direct-wave methods with two station interferometry before correction. For ${ }^{e l l} \mathcal{I}_{3}^{D W}$, the bias is always negative and the absolute bias increases with period. For ${ }^{h y p} \mathcal{I}_{3}^{D W}$, the bias is positive and the absolute bias generally decreases with period.

After the de-biasing correction, the mean and standard deviation of the difference between the $\mathcal{I}_{3}^{D W}$ and $\mathcal{I}_{2}^{A N}$ measurements are shown in Fig. 13d,e and Table 2, which should be contrasted with Fig. 13a,b and Table 1 that contains the same statistics without the de-biasing. The correction decreases the absolute mean difference between the $\mathcal{I}_{3}^{D W}$ and $\mathcal{I}_{2}^{A N}$ measurements at most periods. If we consider the mean difference to be a measure of residual bias, then the bias of the corrected measurements is relatively small $(<5 \mathrm{~m} / \mathrm{s})$ for both $\mathcal{I}_{3}^{D W}$ methods at periods $<40 \mathrm{~s}$. However, the residual bias generally increases at longer periods for both $\mathcal{I}_{3}^{D W}$ methods. Potential causes of and corrections for the residual bias are discussed in section 7.1. 
In contrast with the bias, generally the standard deviations of the differences between the dispersion measurements from the $\mathcal{I}_{2}^{A N}$ method to both $\mathcal{I}_{3}^{D W}$ methods grow with period (Fig. 13a,b and Table 1 (columns 5 \& 7)). Partly, this is due to the decrease in signal-to-noise ratio (SNR) in both the three-station and two-station interferograms at longer periods (Fig. 10a). However, irrespective of SNR, we do not expect the dispersion measurements from the three-station methods to agree with those from the two-station method as well at longer periods. The reason is that the Fresnel Zone or sensitivity kernel for the three-station methods is not identical to the sensitivity kernel for the two-station method and the differences in sensitivity grow with period (section 7.1).

\subsection{Eikonal Tomography}

To further validate and compare the three-station methods we report results from surface wave tomography based on them. To perform tomography, we apply the eikonal tomography method (Lin et al., 2009) to Rayleigh wave phase speed measurements obtained from the two-station and three-station methods. We employ the eikonal tomography method rather than traditional tomographic methods that minimize a penalty functional (e.g., Barmin et al., 2001) because eikonal tomography applies no ad-hoc regularization that depends on data coverage. This simplifies comparison of results from different datasets because they are less affected by differences in the number and distribution of wave paths. In this section, we consider $\mathcal{I}_{3}^{D W}$ only after the de-biasing correction.

The Rayleigh wave phase speed maps produced by the three-station $\left(\mathcal{I}_{3}\right)$ and twostation $\left(\mathcal{I}_{2}^{A N}\right)$ methods are generally quite similar, as displayed at periods of $10 \mathrm{~s}, 20 \mathrm{~s}$, $40 \mathrm{~s}$, and $60 \mathrm{~s}$ in Figs $14-\mathbf{1 7}$. The touchstone is the $\mathcal{I}_{2}^{A N}$ map, and at each period there is substantial agreement between the $\mathcal{I}_{3}$ maps with the $\mathcal{I}_{2}^{A N}$ map. However, we do not show the three-station coda-wave $\left(\mathcal{I}_{3}^{C W}\right)$ maps at periods of $40 \mathrm{~s}$ and $60 \mathrm{~s}$ because the $\mathcal{I}_{3}^{C W}$ method does not provide enough measurements to perform tomography reliably at periods above $30 \mathrm{~s}$. Presumably, this is because the coda is enriched at the shorter periods (Spica et al., 2016; Ansaripour et al., 2019).

A more careful comparison of the tomographic maps requires detailed inspection of the differences between the maps. Let us assume that we have two dispersion maps on the same grid of longitudes $\left(x_{i}\right)$ and latitudes $\left(y_{j}\right): c_{i j}^{(1)}=c^{(1)}\left(x_{i}, y_{j}\right)$ and $c_{i j}^{(2)}=$ 
$c^{(2)}\left(x_{i}, y_{j}\right)$. Let $\Delta_{i j}$ be the difference between these maps:

$$
\Delta_{i j}=c_{i j}^{(1)}-c_{i j}^{(2)},
$$

whose mean over $\left(x_{i}, y_{j}\right)$ is denoted as $\bar{\Delta}$ and standard deviation as $\sigma_{\Delta}$. Figs $18-20$ display such differences between the three-station methods with two-station interferometry in map form and Table 3 summarizes the differences, tabulating $\bar{\Delta}$ and $\sigma_{\Delta}$.

Fig. 18 (and Table 3, column 2) shows the difference between the Rayleigh wave phase speed maps at periods of $10 \mathrm{~s}$ and $20 \mathrm{~s}$ from three-station coda-wave interferometry $\left(\mathcal{I}_{3}^{C W}\right)$ and two-station interferometry $\left(\mathcal{I}_{2}^{A N}\right)$. There is a small systematic difference between the maps $(\bar{\Delta} \approx 7 \mathrm{~m} / \mathrm{s})$ and the standard deviation of the differences is also small $\left(\sigma_{\Delta}<15 \mathrm{~m} / \mathrm{s}\right)$. Unfortunately, we are unable to produce meaningful tomographic maps from $\mathcal{I}_{3}^{C W}$ at longer periods, while it may be more feasible to push $\mathcal{I}_{3}^{C W}$ towards shorter periods than what can be produced by $\mathcal{I}_{2}^{A N}$ (section 7.3 ).

Fig. 19 presents difference maps at periods from $10 \mathrm{~s}$ to $60 \mathrm{~s}$ for the three-station direct-wave method ${ }^{\text {ell }} \mathcal{I}_{3}^{D W}$ relative to $\mathcal{I}_{2}^{A N}$. Table 3, columns 4-5, summarizes the mean and standard deviation of the difference over the maps. The standard deviation of the differences generally grow with period because the $\mathcal{I}_{3}^{D W}$ methods increasingly sample the earth differently than the $\left(\mathcal{I}_{2}^{A N}\right)$ method at longer periods (section 7.1). Larger discrepancies are observed near the peripheries of the maps, where both methods have larger uncertainties. However, the maps are reasonably consistent $\left(\sigma_{\Delta}<25 \mathrm{~m} / \mathrm{s}\right)$ across all periods.

Fig. 20 presents difference maps at periods from $10 \mathrm{~s}$ to $60 \mathrm{~s}$ for the three-station direct-wave method ${ }^{h y p} \mathcal{I}_{3}^{D W}$ relative to $\mathcal{I}_{2}^{A N}$ whose mean and standard deviation are summarized in Table 3, columns 6-7. Similar patterns are observed as in ${ }^{\text {ell }} \mathcal{I}_{3}^{D W}$.

\section{Discussion}

\subsection{Residual Bias of Three-Station Interferometry}

Our de-biasing correction methods are based on straight-ray theory. As shown in section 6, some residual bias exists between three-station direct-wave interferometry $\left(\mathcal{I}_{3}^{D W}\right)$ and two-station interferometry $\left(\mathcal{I}_{2}^{A N}\right)$ even after the correction, especially at the longer periods. We believe this is due to deviation from ray theory. In particular, we dis- 
cuss here the finite frequency effects and the differences in the Fresnel Zones or sensitivity kernels between the methods.

Fig. 21 schematically depicts the difference in sensitivity for the three-station directwave measurements and the two-station measurement, in which we approximate the Fresnel Zone for the two-station method as an ellipse, shown with dashed lines, with the two receiver-stations at the ellipse's foci. The Fresnel Zone for the method ${ }^{e l l} \mathcal{I}_{3}^{D W}$ is approximately the sum of the two Fresnel zones for each of the constituent waves that emanate from the source-station (red dot in Fig. 21a) which lies between the receiver-stations for this method. The sensitivity zone for ${ }^{e l l} \mathcal{I}_{3}^{D W}$ is smaller than for $\mathcal{I}_{2}$, on average, and we therefore expect that the method ${ }^{\text {ell }} \mathcal{I}_{3}^{D W}$ will have a higher resolution than $\mathcal{I}_{2}$, everything else being equal. In contrast, the Fresnel Zone for the method ${ }^{h y p} \mathcal{I}_{3}^{D W}$ is approximately the difference of the two Fresnel zones for each of the constituent waves that emanate from the source-stations (red dots in Fig. 21b), which lie outside the receiverstations. This sensitivity zone for ${ }^{h y p} \mathcal{I}_{3}^{D W}$ is larger and considerably more complicated than for $\mathcal{I}_{2}$, on average. We, therefore, expect that the method ${ }^{h y p} \mathcal{I}_{3}^{D W}$ will have a lower resolution than $\mathcal{I}_{2}$, everything else being equal.

The Fresnel zones for the $\mathcal{I}_{2}^{A N}$ method widen with period, as will those for the $\mathcal{I}_{3}^{D W}$ methods. Therefore, differences between the Fresnel zones of the $\mathcal{I}_{3}^{D W}$ methods compared with the Fresnel zone of the $\mathcal{I}_{2}^{A N}$ method will increase with period, too, as the various methods sample the earth between and around the pair of receiver-stations increasingly differently. We believe this is the source of the increase in the standard deviations of the differences between the phase speed measurements and maps for the various methods (e.g., Fig. 13).

The analysis of Fresnel Zones presented here is schematic and illustrative. The Fresnel Zones have internal structure that will produce details in the sums and differences presented in Fig. 21. General conclusions about the nature of the differences between the various Fresnel Zones are robust, but to use this information quantitatively to improve images in the future will require much more careful computation of the Fresnel zones (e.g., de Vos et al., 2013).

\subsection{Effects of the $26 \mathrm{~s}$ Microseism}

A noteworthy observation is that few reliable measurements exist for three-station coda-wave interferometry $\left(\mathcal{I}_{3}^{C W}\right)$ beyond $40 \mathrm{~s}$ (Fig. 10a). The degradation of quality 
with period for $\mathcal{I}_{3}^{C W}$ is also observed in Spica et al. (2016) and Ansaripour et al. (2019). To understand its cause, we compare the spectra for all methods (Fig. 22). Specifically, we randomly choose 10,000 interferograms from each method and calculate their amplitude spectra. Then the amplitude spectra are normalized and stacked to form mean amplitude spectra curves with standard deviations for each method.

As shown in Fig. 22, the 26 s spatially localized microseism source (e.g., Shapiro et al., 2006; Xia et al., 2013) leaves an imprint on the spectra for all methods although is somewhat stronger for $\mathcal{I}_{3}^{C W}$, which is also indicated in the example FTAN diagrams (Fig. 8). We also compare spectra of $\mathcal{I}_{3}^{C W}$ with and without spectral whitening (Fig. 22de). The whitening makes the spectra flatter at short periods but does not substantially remove the effects of the $26 \mathrm{~s}$ microseism.

The spectra of $\mathcal{I}_{3}^{C W}$ also show much stronger variability at long periods than other methods. Spectral whitening does not help reduce its variability. Thus, although the $26 \mathrm{~s}$ microseism has a stronger effect on $\mathcal{I}_{3}^{C W}$ than other methods, we believe the lack of signals at long periods for $\mathcal{I}_{3}^{C W}$ is largely due to the nature of the coda in two-station interferometry $\left(\mathcal{I}_{2}^{A N}\right)$.

\subsection{Potential for Further Refinement}

We have chosen many of the characteristics of the two-station and three-station interferometric methods in a reasoned but largely ad-hoc way. Thus, all of the procedures we describe above may be refined to improve some aspect of the results. Such refinements could be made (1) to the data processing procedures, (2) to the definition of the stationary phase zones for the direct-wave methods, (3) to the de-biasing procedure applied to the direct-wave methods, and (4) to the use of the results from the different methods in concert with one another.

(1) Data processing procedures include the definition of both the direct-wave and coda-wave windows, the wavelength criterion for the minimum inter-receiver-station distance, the chosen values of the stacking weights $w_{j}$, and the use of only the symmetric component of the two-station ambient noise interferograms as the basis for all of data processing. In addition, the two-station data processing procedures of Shen and Ritzwoller (2016) underlie our results, including the use of an 80 s moving average time-domain normalization window and spectral whitening. All of these choices may be revised in the 
future to optimize the result of data processing. For three-station coda-wave interferometry $\left(\mathcal{I}_{3}^{C W}\right)$, performing interferometry on hourly or daily $\mathcal{I}_{2}$ and then stack hourly or daily $\mathcal{I}_{3}^{C W}$ (Zhang \& Yang, 2013; Haendel et al., 2016) may greatly increase SNR because cross-talk between incoherent asynchronous signals are avoided (Sheng et al., 2018). Despite its inapplicability to asynchronous pairs, this pre-stacking scheme may be promising for extraction of short-period information (Sheng et al., 2018).

(2) Another important characteristic of the three-station direct-wave methods is the definition of the stationary-phase zones. We choose $\alpha=10^{-2}$ in eqs. (2) and (3) to be period-independent, which produces a maximum angle of both the elliptical and hyperbolic stationary phase zones of about $\theta=8^{\circ}$. An optimal period-dependent parameterization of the stationary phase zones may be possible. Moreover, because increasing $\alpha$ should increase the bias of the three-station methods, in station-rich settings $\alpha$ may be reduced and in station-poor regions it may be increased, although at the expense of increasing bias.

(3) The de-biasing method outlined in section 5 applies corrections to dispersion curves before they are statistically summarized for each path, based on a great-circle raytheoretic procedure. This method could be improved by correcting additional errors from off-great-circle propagation (Yao et al., 2006; Foster et al., 2014), non-plane waves (Pedersen, 2006) and finite-frequency effects (Yao et al., 2010; de Vos et al., 2013). Alternately, a completely different approach may be possible, which applies phase corrections to sourcespecific interferograms $\left(C_{3}^{D W}\right)$ and then makes the dispersion measurements on the composite Green's function $\left(\hat{G}_{3}\right)$. The correction is a (frequency-dependent) phase shift to each of the source-specific interferograms prior to stacking.

(4) Because the three-station methods $\left(\mathcal{I}_{3}\right)$ are consistent with the two-station method $\left(\mathcal{I}_{2}\right)$, measurements from all methods can be combined simultaneously. It might be particularly advantageous to combine measurements from methods ${ }^{\text {ell }} \mathcal{I}_{3}^{D W}$ and ${ }^{\text {hyp }} \mathcal{I}_{3}^{D W}$ because they are oppositely biased, and their biases may cancel approximately without an explicit bias correction.

\subsection{Connections to Other Methods}

We now discuss how three-station interferometry $\left(\mathcal{I}_{3}\right)$ methods connect to and differ from other interferometric methods, and how the methods can gain insight from each 
other. In particular, we discuss the earthquake two-station method (Sato, 1955), sourcereceiver interferometry (Curtis \& Halliday, 2010), and generalized interferometry not based on Green's function retrieval (Fichtner et al., 2017).

In the earthquake two-station method, for an earthquake lying approximately inline with two receivers, seismograms recorded at two receivers are correlated to extract information about the inter-receiver medium (e.g., Landisman et al., 1969). Thus, if the earthquake is replaced by a station and the earthquake seismograms are replaced by interstation noise correlations, then the configuration of the earthquake two-station method is somewhat similar to three-station direct-wave interferometry method ${ }^{h y p} \mathcal{I}_{3}^{D W}$. In this study, source-receiver distances are similar in scale to inter-receiver distances while global earthquakes are often used for regional studies in the earthquake two-station method (e.g., Yao et al., 2006). Thus, global source-stations may also be used in $\mathcal{I}_{3}^{D W}$ which may provide longer period information in the future.

Source-receiver interferometry (SRI; Curtis \& Halliday, 2010) presents three types of geometries where one can extract the Green's function between a source and a receiver without direct observation: correlation-correlation SRI, correlation-convolution SRI, and convolution-convolution SRI. The geometries of three-station direct-wave interferometry methods ${ }^{h y p} \mathcal{I}_{3}^{D W}$ and ${ }^{e l l} \mathcal{I}_{3}^{D W}$ are similar to the correlation-correlation SRI and correlationconvolution SRI, respectively, with a station serving as a virtual source. A critical difference between three-station interferometry and SRI is that our goal is to obtain reliable dispersion measurements from the direct Rayleigh waves, which requires us to resolve the source phase $\phi_{s}$ (section 4.1). The tapering of stationary phase zones and the area weights of source-stations in Entwistle et al. (2015) will affect $\phi_{s}$ in a complicated way, so that the tapering and the area weights are not used here. When amplitude information can be reliably interpreted from two-station interferograms $\left(\mathcal{I}_{2}\right)$, the tapering and the area weights may provide a closer approximation to the theoretical integral of Green's function retrieval and thus might also benefit phase measurements.

Finally, the three-station direct-wave methods $\left(\mathcal{I}_{3}^{D W}\right)$ could work optimally for new generalized interferometric methods not based on estimating Green's functions. New methods of interferometry are being developed that attempt to extract information about the sources and propagating medium jointly irrespective of the relative position of the sources and receiver-stations (e.g., Tromp et al., 2010; Hanasoge, 2014; Fichtner et al., 2017; Sager 
et al., 2018). $\mathcal{I}_{3}^{D W}$, where the location of source-stations is known exactly, may provide an ideal application for these methods.

\section{Conclusions}

Our principal finding is that the three-station direct-wave interferometry methods ${ }^{e l l} \mathcal{I}_{3}^{D W}$ and ${ }^{h y p} \mathcal{I}_{3}^{D W}$ generally outperform three-station coda-wave interferometry $\mathcal{I}_{3}^{C W}$ for obtaining Rayleigh wave dispersion measurements, even though direct-wave interferometry has been largely ignored as an imaging tool to date. This outperformance includes such metrics as signal-to-noise ratio, the number of measurements returned, and most notably the band-width of the measurements because $\mathcal{I}_{3}^{C W}$ is primarily confined to providing measurements below 25 s period. In addition, the direct-wave methods also outperform two-station interferometry in these metrics.

There are two primary caveats concerning the performance of the three-station directwave methods. First, the ${ }^{e l l} \mathcal{I}_{3}^{D W}$ and ${ }^{h y p} \mathcal{I}_{3}^{D W}$ methods are slightly biased relative to twostation interferometry, $\mathcal{I}_{2}^{A N}$. However, we present a ray-theoretic de-biasing procedure that nearly eliminates the bias at and below about $40 \mathrm{~s}$ period, where ray-theory is expected to work best, and substantially reduces bias at longer periods. Second, the sensitivity kernels for the three-station direct-wave methods are more complicated than both two-station interferometry and three-station coda-wave interferometry and remain poorly understood. Research is needed to understand the nature of the sensitivity kernels for the three-station direct-wave methods and how they compare to two-station interferometry.

The tests presented here use data from the EarthScope Transportable Array (TA), but the relative merits of the various methods tested may vary in different settings where station coverage and geometries will differ. Indeed, the three-station methods that we test here may be least needed in the contiguous US due to the outstanding data coverage provided by the TA. Tests in different regions (e.g., Antarctica, Tibet, Europe, Alaska, the Juan de Fuca Plate, etc.) are needed to determine how the methods will perform in a variety of settings.

Irrespective of these caveats, we believe that three-station direct-wave interferometry promises to provide a substantial new tool to the toolbox of standard methods for imaging the structure of the crust and uppermost mantle. We encourage seismologists 
to bear in mind its ability to bridge asynchronously deployed stations in designing new seismic networks.

\section{Acknowledgments}

We thank Andrew Curtis, an anonymous reviewer and the editor, Sidao Ni, for constructive comments. We are grateful to Weisen Shen for providing noise correlations. GMT (Wessel et al., 2013) and ObsPy (Beyreuther et al., 2010) are used in data processing. Source codes for this project are available on GitHub (https://github.com/NoiseCIEI/ ThreeStation). This research was supported in part by NSF grants EAR-1537868, EAR1645269, and EAR-1928395 at the University of Colorado at Boulder. The facilities of IRIS Data Services, and specifically the IRIS Data Management Center, were used for access to waveforms, related metadata and/or derived products used in this study. IRIS Data Services are funded through the Seismological Facilities for the Advancement of Geoscience and EarthScope (SAGE) Proposal of the National Science Foundation under Cooperative Agreement EAR-1261681. This work utilized the RMACC Summit supercomputer, which is supported by the National Science Foundation (awards ACI-1532235 and ACI-1532236), the University of Colorado Boulder, and Colorado State University. The Summit supercomputer is a joint effort of the University of Colorado Boulder and Colorado State University.

\section{References}

Ansaripour, M., Rezapour, M., \& Saygin, E. (2019). Shear wave velocity structure of Iranian Plateau: Using combination of ambient noise cross-correlations $\left(C^{1}\right)$ and correlation of coda of correlations $\left(C^{3}\right) . \quad$ Geophysical Journal International, ggz257. doi: 10.1093/gji/ggz257

Barmin, M. P., Ritzwoller, M. H., \& Levshin, A. L. $\quad$ (2001). $\quad$ A fast and reliable method for surface wave tomography. $\quad$ Pure and Applied Geophysics, 158(8), 1351-1375. doi: 10.1007/978-3-0348-8264-4_3

Bensen, G. D., Ritzwoller, M. H., Barmin, M. P., Levshin, A. L., Lin, F., Moschetti, M. P., ... Yang, Y. (2007). Processing seismic ambient noise data to obtain reliable broad-band surface wave dispersion measurements. Geophysical Journal International, 169(3), 1239-1260. doi: 10.1111/j.1365-246X.2007.03374.x

Beyreuther, M., Barsch, R., Krischer, L., Megies, T., Behr, Y., \& Wassermann, J. 
(2010). ObsPy: A Python toolbox for seismology. Seismological Research Letters, 81(3), 530-533. doi: 10.1785/gssrl.81.3.530

Curtis, A., Behr, Y., Entwistle, E., Galetti, E., Townend, J., \& Bannister, S. (2012). The benefit of hindsight in observational science: Retrospective seismological observations. $\quad$ Earth and Planetary Science Letters, 345-348, 212-220. doi: 10.1016/j.epsl.2012.06.008

Curtis, A., \& Halliday, D. (2010). Source-receiver wave field interferometry. Physical Review E, 81(4), 046601. doi: 10.1103/PhysRevE.81.046601

de Vos, D., Paulssen, H., \& Fichtner, A. (2013). Finite-frequency sensitivity kernels for two-station surface wave measurements. Geophysical Journal International, 194(2), 1042-1049. doi: 10.1093/gji/ggt144

Duguid, C., Halliday, D., \& Curtis, A. (2011). Source-receiver interferometry for seismic wavefield construction and ground-roll removal. The Leading Edge, 30(8), 838-843. doi: 10.1190/1.3626489

Dziewonski, A., Bloch, S., \& Landisman, M. (1969). A technique for the analysis of transient seismic signals. Bulletin of the Seismological Society of America, $59(1), 427-444$.

Entwistle, E., Curtis, A., Galetti, E., Baptie, B., \& Meles, G. (2015). Constructing new seismograms from old earthquakes: Retrospective seismology at multiple length scales. Journal of Geophysical Research: Solid Earth, 120(4), 2466-2490. doi: 10.1002/2014JB011607

Fenneman, N. M., \& Johnson, D. W. (1946). Physical divisions of the United States. Reston, VA: US Geological Survey.

Fichtner, A., Stehly, L., Ermert, L., \& Boehm, C. (2017). Generalised interferometry - I: Theory for inter-station correlations. Geophysical Journal International, 208(2), 603-638. doi: 10.1093/gji/ggw420

Foster, A., Ekström, G., \& Nettles, M. (2014). Surface wave phase velocities of the Western United States from a two-station method. Geophysical Journal International, 196(2), 1189-1206. doi: 10.1093/gji/ggt454

Froment, B., Campillo, M., \& Roux, P. (2011). Reconstructing the Green's function through iteration of correlations. Comptes Rendus Geoscience, 343(8-9), 623632. doi: $10.1016 /$ j.crte.2011.03.001

Garnier, J., \& Papanicolaou, G. (2009). Passive sensor imaging using cross correla- 
tions of Noisy Signals in a scattering medium. SIAM Journal on Imaging Sciences, 2(2), 396-437. doi: 10.1137/080723454

Haendel, A., Ohrnberger, M., \& Krüger, F. (2016). Extracting near-surface $Q_{\mathrm{L}}$ between $1-4 \mathrm{~Hz}$ from higher-order noise correlations in the Euroseistest area, Greece. Geophysical Journal International, 207(2), 655-666. doi: $10.1093 / \mathrm{gji} / \mathrm{ggw} 295$

Halliday, D., \& Curtis, A. (2009). Seismic interferometry of scattered surface waves in attenuative media. Geophysical Journal International, 178(1), 419-446. doi: 10.1111/j.1365-246X.2009.04153.x

Hanasoge, S. M. (2014). Measurements and kernels for source-structure inversions in noise tomography. Geophysical Journal International, 196(2), 971-985. doi: 10 $.1093 /$ gji/ggt411

Landisman, M., Dziewonski, A., \& Satô, Y. (1969). Recent improvements in the analysis of surface wave observations. Geophysical Journal International, 17(4), 369-403. doi: 10.1111/j.1365-246X.1969.tb00246.x

Levshin, A. L., \& Ritzwoller, M. H. (2001). Automated detection, extraction, and measurement of regional surface waves. Pure and Applied Geophysics, 158(8), 1531-1545. doi: 10.1007/978-3-0348-8264-4_11

Lin, F.-C., Moschetti, M. P., \& Ritzwoller, M. H. (2008). Surface wave tomography of the western United States from ambient seismic noise: Rayleigh and Love wave phase velocity maps. Geophysical Journal International, 173(1), 281-298. doi: $10.1111 / \mathrm{j} .1365-246 \mathrm{X} .2008 .03720 . \mathrm{x}$

Lin, F.-C., Ritzwoller, M. H., \& Snieder, R. (2009). Eikonal tomography: Surface wave tomography by phase front tracking across a regional broad-band seismic array. Geophysical Journal International, 177(3), 1091-1110. doi: 10.1111/j.1365-246X.2009.04105.x

Ma, S., \& Beroza, G. C. (2012). Ambient-field Green's functions from asynchronous seismic observations. Geophysical Research Letters, 39(6), L06301. doi: 10 $.1029 / 2011$ GL050755

Pedersen, H. A. (2006). Impacts of non-plane waves on two-station measurements of phase velocities. Geophysical Journal International, 165(1), 279-287. doi: 10 $.1111 / \mathrm{j} .1365-246 X .2006 .02893 . \mathrm{x}$

Ritzwoller, M. H., \& Feng, L. (2019). Overview of pre- and post-processing of 
ambient-noise correlations.

Seismic Ambient Noise. $10.1017 / 9781108264808$

In N. Nakata, L. Gualtieri, \& A. Fichtner (Eds.), Cambridge: Cambridge University Press. doi:

Sabra, K. G., Gerstoft, P., Roux, P., Kuperman, W. A., \& Fehler, M. C.

(2005). Surface wave tomography from microseisms in Southern California. Geophysical Research Letters, 32(14), L14311. doi: 10.1029/2005GL023155

Sager, K., Ermert, L., Boehm, C., \& Fichtner, A. (2018). Towards full waveform ambient noise inversion. Geophysical Journal International, 212(1), 566-590. doi: $10.1093 /$ gji $/ \operatorname{ggx} 429$

Sato, Y. (1955). Analysis of dispersed surface waves by means of Fourier transform I. Bull. Earthq. Res. Inst., Univ. Tokyo, 33, 33-48.

Shapiro, N. M., \& Campillo, M. (2004). Emergence of broadband Rayleigh waves from correlations of the ambient seismic noise. Geophysical Research Letters, 31(7), L07614. doi: 10.1029/2004GL019491

Shapiro, N. M., Campillo, M., Stehly, L., \& Ritzwoller, M. H. (2005). Highresolution surface-wave tomography from ambient seismic noise. Science, 307(5715), 1615-1618. doi: 10.1126/science.1108339

Shapiro, N. M., Ritzwoller, M. H., \& Bensen, G. D. (2006). Source location of the 26 sec microseism from cross-correlations of ambient seismic noise. Geophysical Research Letters, 33(18), L18310. doi: 10.1029/2006GL027010

Shen, W., \& Ritzwoller, M. H. (2016). Crustal and uppermost mantle structure beneath the United States. Journal of Geophysical Research: Solid Earth, 121(6), 4306-4342. doi: 10.1002/2016JB012887

Sheng, Y., Denolle, M. A., \& Beroza, G. C. ～(2017). Multicomponent C3 Green's functions for improved long-period ground-motion prediction. Bulletin of the Seismological Society of America, 107(6), 2836-2845. doi: $10.1785 / 0120170053$

Sheng, Y., Nakata, N., \& Beroza, G. C. (2018). On the nature of higher-order ambient seismic field correlations. Journal of Geophysical Research: Solid Earth, 123(9), 7969-7982. doi: 10.1029/2018JB015937

Slob, E., \& Wapenaar, K. (2007). Electromagnetic Green's functions retrieval by cross-correlation and cross-convolution in media with losses. Geophysical Research Letters, 34(5), L05307. doi: 10.1029/2006GL029097 
Snieder, R. (2004). Extracting the Green's function from the correlation of coda waves: A derivation based on stationary phase. Physical Review E, 69(4), 046610. doi: 10.1103/PhysRevE.69.046610

Spica, Z., Perton, M., \& Beroza, G. C. (2017). Lateral heterogeneity imaged by small-aperture $S c S$ retrieval from the ambient seismic field. Geophysical Research Letters, 44(16), 8276-8284. doi: 10.1002/2017GL073230

Spica, Z., Perton, M., Calò, M., Legrand, D., Córdoba-Montiel, F., \& Iglesias, A. (2016). 3-D shear wave velocity model of Mexico and South US: Bridging seismic networks with ambient noise cross-correlations $\left(C^{1}\right)$ and correlation of coda of correlations $\left(C^{3}\right)$. Geophysical Journal International, 206(3), 17951813. doi: $10.1093 /$ gji/ggw240

Stehly, L., Campillo, M., Froment, B., \& Weaver, R. L. (2008). Reconstructing Green's function by correlation of the coda of the correlation $\left(C^{3}\right)$ of ambient seismic noise. Journal of Geophysical Research, 113(B11), B11306. doi: 10.1029/2008JB005693

Tromp, J., Luo, Y., Hanasoge, S., \& Peter, D. (2010). Noise cross-correlation sensitivity kernels. Geophysical Journal International, 183(2), 791-819. doi: 10 $.1111 / \mathrm{j} .1365-246 \mathrm{X} .2010 .04721 . \mathrm{x}$

Tsai, V. C. (2009). On establishing the accuracy of noise tomography travel-time measurements in a realistic medium. Geophysical Journal International, 178(3), 1555-1564. doi: 10.1111/j.1365-246X.2009.04239.x

Wessel, P., Smith, W. H. F., Scharroo, R., Luis, J., \& Wobbe, F. (2013). Generic Mapping Tools: Improved version released. Eos, Transactions American Geophysical Union, 94(45), 409-410. doi: 10.1002/2013EO450001

Xia, Y., Ni, S., \& Zeng, X. (2013). Twin enigmatic microseismic sources in the Gulf of Guinea observed on intercontinental seismic stations. Geophysical Journal International, 194(1), 362-366. doi: 10.1093/gji/ggt076

Yao, H., \& van der Hilst, R. D. (2009). Analysis of ambient noise energy distribution and phase velocity bias in ambient noise tomography, with application to SE Tibet. Geophysical Journal International, 179(2), 1113-1132. doi: 10.1111/j.1365-246X.2009.04329.x

Yao, H., van der Hilst, R. D., \& de Hoop, M. V. (2006). Surface-wave array tomography in SE Tibet from ambient seismic noise and two-station analysis - I. 
phase velocity maps. Geophysical Journal International, 166(2), 732-744. doi: $10.1111 / \mathrm{j} .1365-246 \mathrm{X} .2006 .03028 . \mathrm{x}$

Yao, H., van der Hilst, R. D., \& Montagner, J.-P. (2010). Heterogeneity and anisotropy of the lithosphere of SE Tibet from surface wave array tomography. Journal of Geophysical Research: Solid Earth, 115(B12), B12307. doi: 10.1029/2009JB007142

Zhang, J., \& Yang, X. (2013). Extracting surface wave attenuation from seismic noise using correlation of the coda of correlation. Journal of Geophysical Research: Solid Earth, 118(5), 2191-2205. doi: 10.1002/jgrb.50186 
Table 1. Differences $(\mathrm{m} / \mathrm{s})$ of Rayleigh wave phase speed measurements from the $\mathcal{I}_{3}$ methods compared to $\mathcal{I}_{2}$ before the de-biasing correction.

\begin{tabular}{crrrrrr}
\hline & \multicolumn{2}{c}{$\mathcal{I}_{3}^{C W}$} & \multicolumn{2}{c}{${ }^{\text {ell } \mathcal{I}_{3}{ }^{D W}}$} & ${ }^{h y p} \mathcal{I}_{3}{ }^{D W}$ \\
Period (s) & Mean & SD & Mean & SD & Mean & SD \\
\hline 10 & 0.1 & 7.3 & -9.3 & 13.5 & 11.5 & 14.1 \\
20 & -0.0 & 17.0 & -9.9 & 13.8 & 13.3 & 12.9 \\
30 & -1.4 & 43.2 & -9.9 & 18.4 & 14.0 & 18.2 \\
40 & - & - & -8.1 & 24.1 & 10.8 & 23.6 \\
50 & - & - & -11.4 & 27.2 & 10.4 & 27.5 \\
60 & - & - & -12.8 & 27.0 & 8.3 & 28.8 \\
70 & - & - & -14.7 & 25.8 & 5.0 & 28.4 \\
80 & - & - & -15.0 & 24.2 & 0.6 & 28.8 \\
\hline
\end{tabular}

Table 2. Differences $(\mathrm{m} / \mathrm{s})$ of Rayleigh wave phase speed measurements from the direct-wave $\mathcal{I}_{3}$ methods compared to $\mathcal{I}_{2}$ after the de-biasing correction.

\begin{tabular}{crrrr}
\hline & ${ }^{e l l} \mathcal{I}_{3}^{D W}$ & ${ }^{\text {hyp }} \mathcal{I}_{3}^{D W}$ \\
Period (s) & Mean & SD & Mean & SD \\
\hline 10 & 0.6 & 5.5 & -1.3 & 16.8 \\
20 & 0.3 & 7.2 & 1.7 & 14.0 \\
30 & -0.9 & 11.5 & 3.4 & 19.4 \\
40 & -1.7 & 17.7 & 3.8 & 26.7 \\
50 & -7.6 & 24.2 & 8.1 & 31.7 \\
60 & -5.9 & 28.0 & 6.4 & 34.3 \\
70 & -6.7 & 28.4 & 4.5 & 35.2 \\
80 & -4.7 & 27.4 & 2.2 & 34.4 \\
\hline
\end{tabular}


Table 3. Differences $(\mathrm{m} / \mathrm{s})$ of Rayleigh wave phase speed maps from the $\mathcal{I}_{3}$ methods compared to $\mathcal{I}_{2}$ after the de-biasing correction.

\begin{tabular}{|c|c|c|c|c|c|c|}
\hline \multirow[b]{2}{*}{ Period (s) } & \multicolumn{2}{|c|}{$\mathcal{I}_{3}^{C W}$} & \multicolumn{2}{|c|}{${ }^{\text {ell }} \mathcal{I}_{3}^{D W}$} & \multicolumn{2}{|c|}{${ }^{\text {hyp }} \mathcal{I}_{3}^{D W}$} \\
\hline & Mean & $\mathrm{SD}$ & Mean & SD & Mean & SD \\
\hline 10 & 7.0 & 11.8 & -0.8 & 8.1 & -2.9 & 8.7 \\
\hline 20 & 7.4 & 13.8 & -1.8 & 5.2 & -0.5 & 5.6 \\
\hline 30 & - & - & 1.9 & 8.8 & 1.0 & 7.0 \\
\hline 40 & - & - & -0.4 & 12.3 & 0.8 & 15.4 \\
\hline 50 & - & - & 9.9 & 16.2 & 2.1 & 17.9 \\
\hline 60 & - & - & 1.2 & 24.1 & 6.5 & 23.3 \\
\hline
\end{tabular}




\section{(b) Three-Station Interferometry}

\section{(a) Two-Station Interferometry}
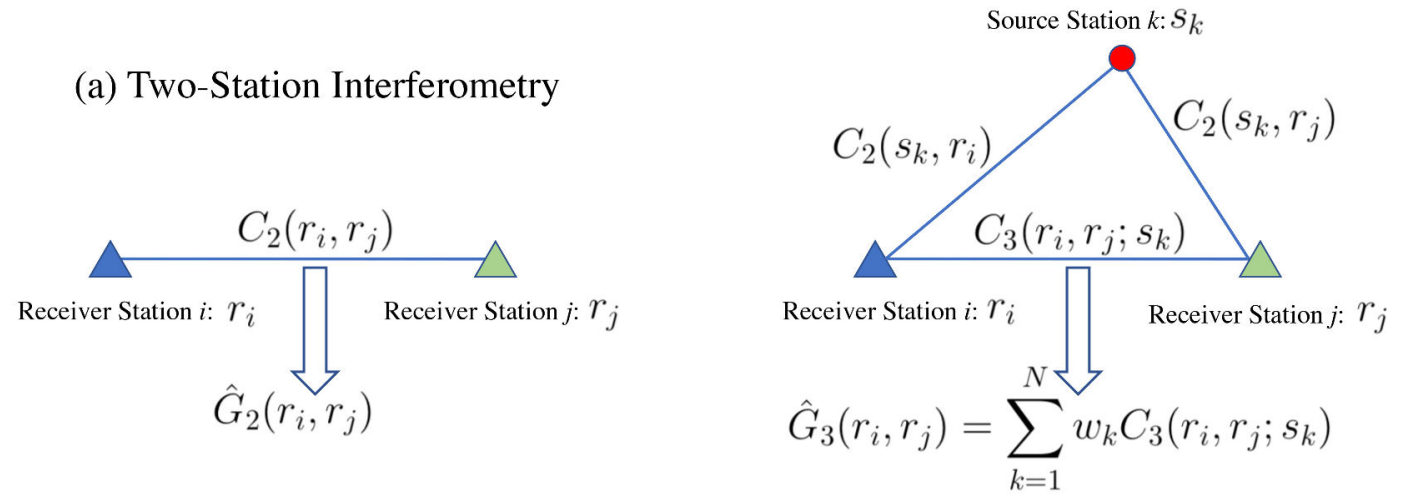

Figure 1. Notation for interferometry. (a) Two-station interferometry. $C_{2}\left(r_{i}, r_{j}\right)$ is the cross-correlation between processed seismograms recorded at receiver-stations $r_{i}$ and $r_{j}$. The twostation estimated Green's function, $\hat{G}_{2}\left(r_{i}, r_{j}\right)$, can be determined from $C_{2}$ after applying an appropriate phase shift. Receiver-stations $r_{i}$ and $r_{j}$ must operate synchronously. (b) Three-station interferometry. Cross-correlations between seismograms recorded at each source-station, $s_{k}$, with records at receiver-stations, $r_{i}$ and $r_{j}$, are denoted $C_{2}\left(s_{k}, r_{i}\right)$ and $C_{2}\left(s_{k}, r_{j}\right)$. Direct-wave or coda-wave parts of these records are cross-correlated or convolved to measure the source-specific interferogram, $C_{3}\left(r_{i}, r_{j} ; s_{k}\right)$, which can be summed over contributions from many source-stations to produce the three-station composite Green's function, $\hat{G}_{3}\left(r_{i}, r_{j}\right)$, between the receiver-stations. Receiver-stations $r_{i}$ and $r_{j}$ need not operate synchronously with one another, but both must overlap the operation of each source-station. 


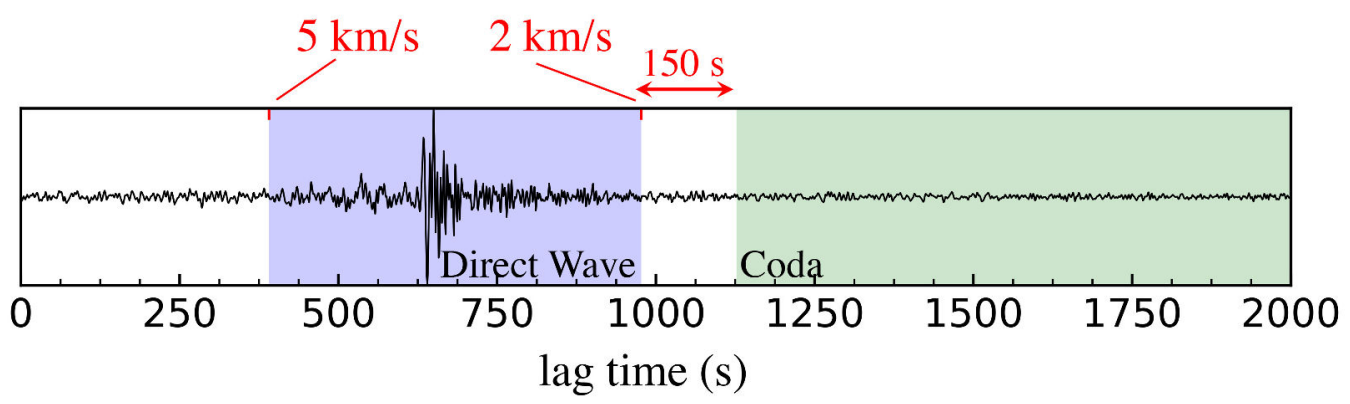

Figure 2. Example of the definition of the direct-wave and coda-wave segments of a twostation cross-correlation of ambient noise, $C_{2}$, for stations ANMO (Albuquerque, NM) and M47A (Cromwell, IN), at an inter-station distance of $\sim 1950 \mathrm{~km}$. The direct-wave is the segment of the record between times corresponding to group speeds of 2 and $5 \mathrm{~km} / \mathrm{s}$. The coda-wave segment starts $150 \mathrm{~s}$ after the end of the direct-wave, and extends to the end of $3000 \mathrm{~s}$. The symmetric component of the cross-correlation is shown (average of positive and negative correlation lags).
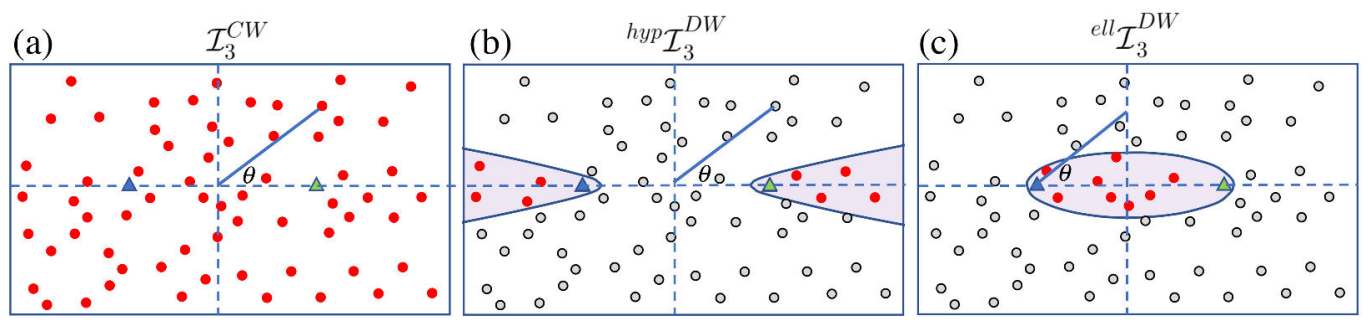

Figure 3. Schematic illustration of the geometrical constraints on source-stations for different methods of three-station interferometry. The two receiver-stations are shown with the bue and green triangles, and the circles are locations of other stations that may act as source-stations.

Those stations that can act as source-stations are shown with red circles and those cannot with grey circles. (a) For three-station coda-wave interferometry, $\mathcal{I}_{3}^{C W}$, all stations whose operation overlaps the two receiver-stations can act as a source-station. (b) For three-station direct-wave interferometry with source-stations radially outside the receiver-stations, ${ }^{h y p} \mathcal{I}_{3}^{D W}$, source-stations must lie in stationary phase hyperbolae (purple shading). (c) For three-station direct-wave interferometry with source-stations between the receiver-stations, ${ }^{\text {ell }} \mathcal{I}_{3}^{D W}$, source-stations must lie in the stationary phase ellipse (purple shading). The angle $\theta$ in each case is defined in section 3.1 and used in Fig. 5 . 
(a)

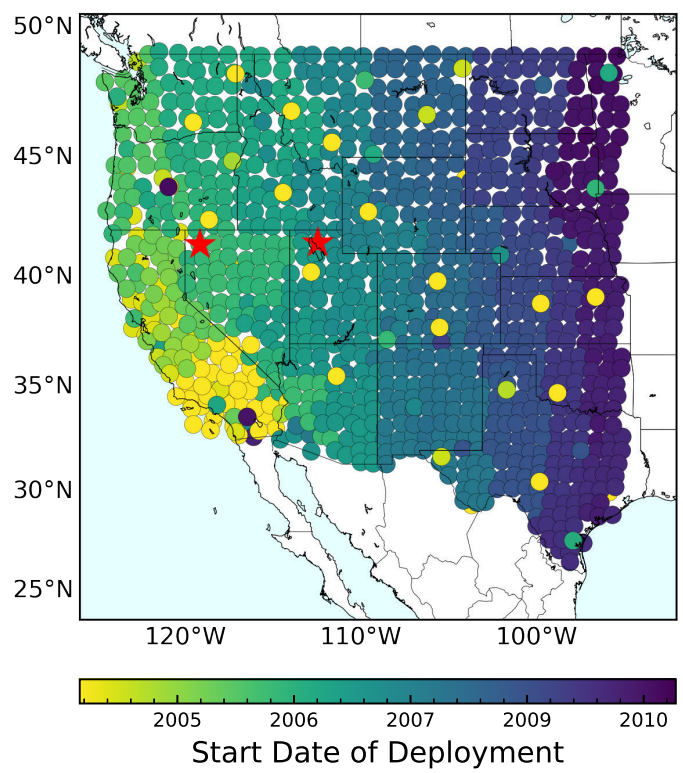

(b)

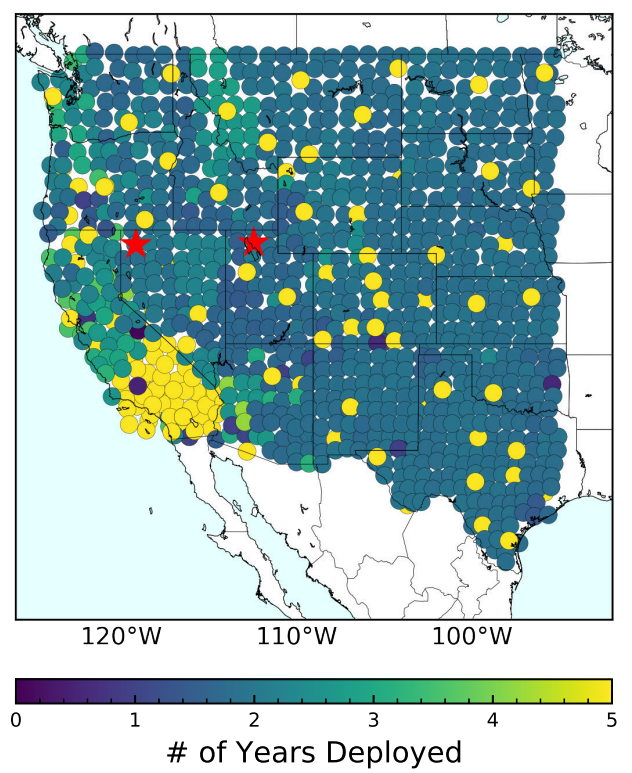

Figure 4. Map of stations used in this study. Red stars mark stations used in Figs 5, 6 and 8: M07A (Soldier Meadow, NV) and M15A (Promontory, UT). (a) The start dates for each station are color-coded, showing a rolling pattern from west to east. (b) Duration of deployment is color-coded. Most stations are deployed around two years with a few much longer from the USArray Reference Network (_US-REF) and the Southern California Seismic Network (CI). 

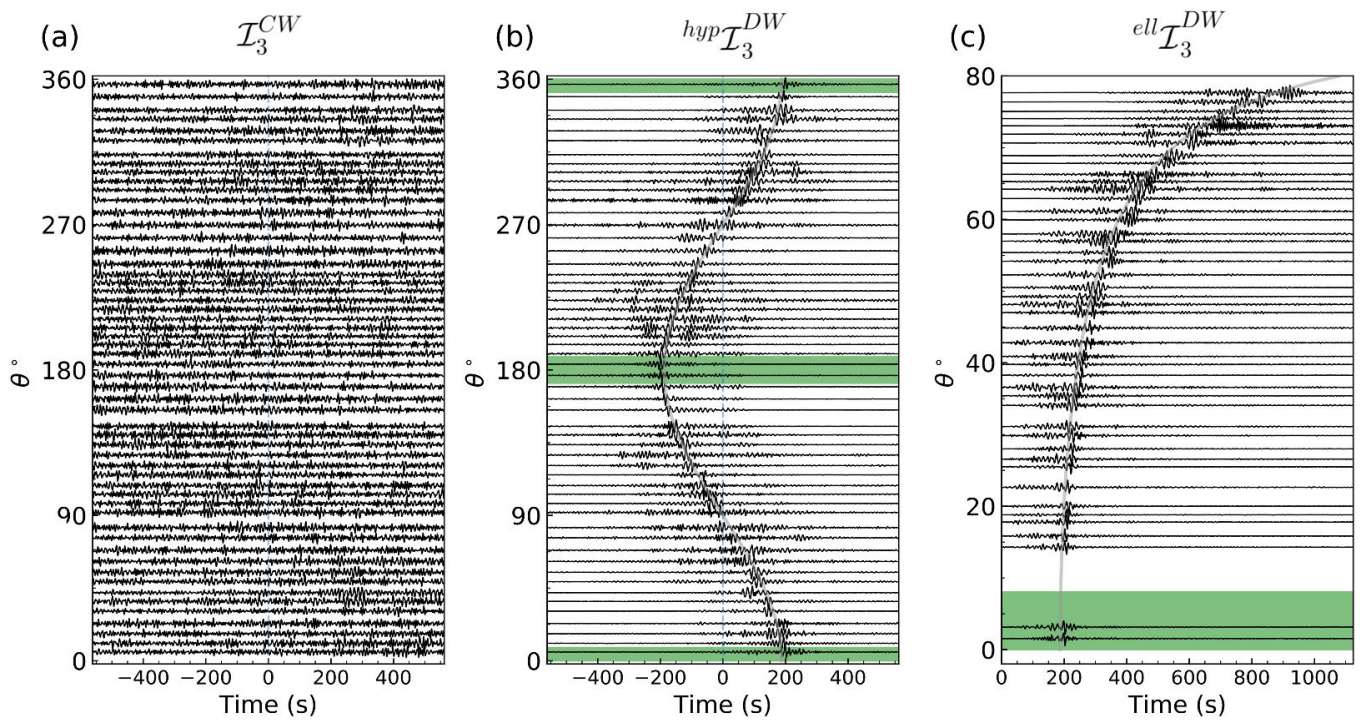

Figure 5. Example record sections of three-station interferograms for the receiver-station pair M07A-M15A, whose locations are shown in Fig. 4 . (a) Coda-wave correlations $\left(C_{3}^{C W}\right)$ for different source-stations plotted at the azimuth angle $\theta$ shown in Fig. 3a. (b) Direct-wave correlations $\left(C_{3}^{D W}\right)$ plotted for source-stations at the azimuth angle shown in Fig. $\mathbf{3 b}$. The green regions are the hyperbolic stationary-phase zones for ${ }^{h y p} \mathcal{I}_{3}^{D W}$. (c) Direct-wave convolutions $\left(C_{3}^{D W}\right)$ plotted for source-stations at the azimuth angle shown in Fig. 3c. Only positive time lags are defined. The green region is the elliptical stationary-phase zone for ${ }^{e l l} \mathcal{I}_{3}^{D W}$. Grey curves in (b) and (c) are predictions from eqs. (4) and (5), respectively, with $c=3 \mathrm{~km} / \mathrm{s}$. Only selected three-station interferograms are shown to ease visualization. 
(a) $\mathcal{I}_{3}^{C W}$

M07A - M15A

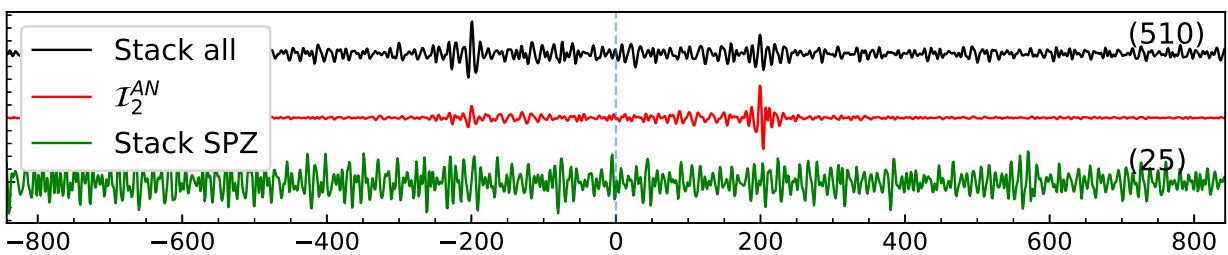

(b) ${ }^{\text {hyp }} \mathcal{I}_{3}^{D W}$

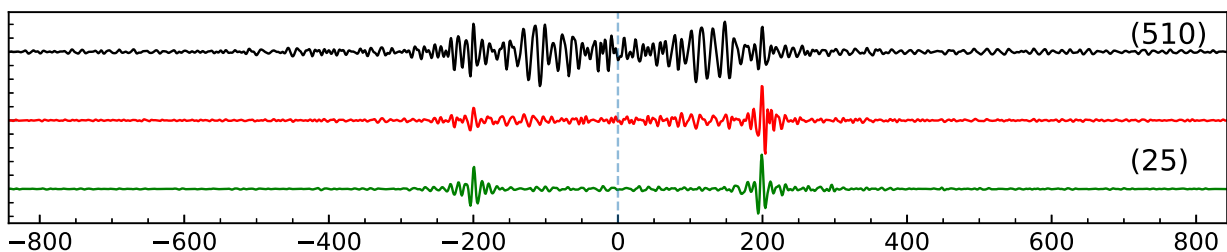

(c) ${ }^{\text {ell }} \mathcal{I}_{3}^{D W}$

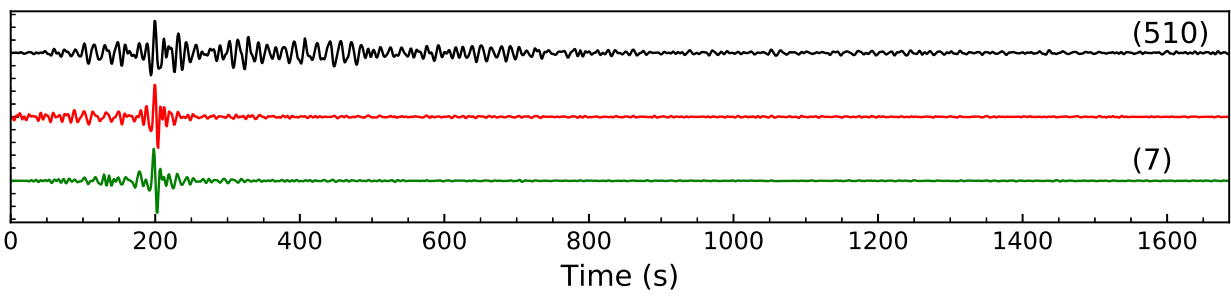

Figure 6. Examples of stacks of three-station interferograms for the receiver-station pair M07A-M15A of Fig. 5. In each panel the two-station estimated Green's function $\left(\mathcal{I}_{2}^{A N}\right)$ is plotted for reference (red). The number of source-stations for each stack is shown in parentheses above the stacked trace. (a) Method $\mathcal{I}_{3}^{C W}$. Two stacks of coda-wave interferograms are shown: (black line) stack of the interferograms from all source-stations irrespective of the azimuthal angle $\theta$ (defined in Fig. 3a) and (green line) stack of the coda-wave interferograms for sources in the hyperbolic stationary phase zone. For $\mathcal{I}_{3}^{C W}$, the black line is the composite Green's function. (b) Method ${ }^{h y p} \mathcal{I}_{3}^{D W}$. Black and green lines have similar meanings to those in (a), but here the direct-wave interferograms are stacked. For ${ }^{h y p} \mathcal{I}_{3}^{D W}$, the green line is the composite Green's function. (c) Method ${ }^{e l l} \mathcal{I}_{3}^{D W}$. Black line is the same as in (b), but the green line is the stack of direct-wave interferograms in the elliptical stationary phase zone. For ${ }^{e l l} \mathcal{I}_{3}^{D W}$, the green line is the composite Green's function and only positive time lags are defined. 
(a)

$$
{ }^{e l l} \mathcal{I}_{3}^{D W}
$$

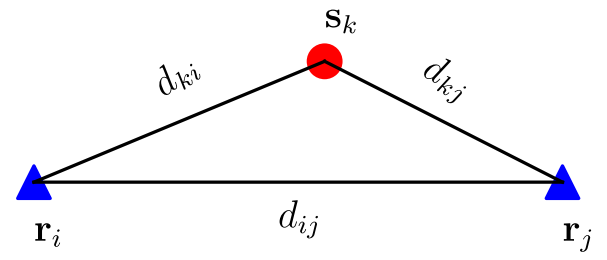

(b)

$$
{ }^{h y p} \mathcal{I}_{3}^{D W}
$$

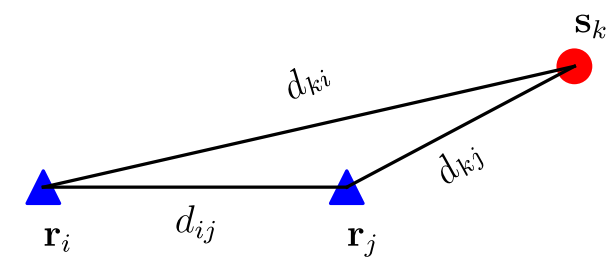

Figure 7. Geometry of the source-station $\left(s_{k}\right)$ and receiver-stations $\left(r_{i}, r_{j}\right)$ used to determine the phase for the three-station direct-wave methods: (a) ${ }^{e l l} \mathcal{I}_{3}^{D W}$ and (b) ${ }^{\text {hyp }} \mathcal{I}_{3}^{D W}$. Great circle distances between two stations are denoted as $d$ with appropriate subscripts.

(a) $\mathcal{I}_{2}^{A N}$

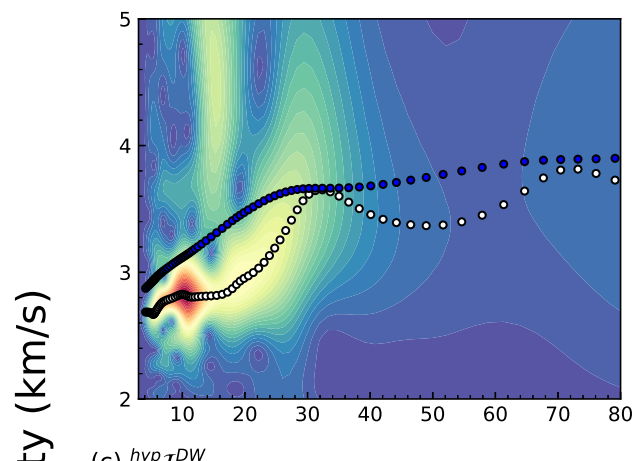

(c) ${ }^{\text {hyp }} \mathcal{I}_{3}^{D W}$

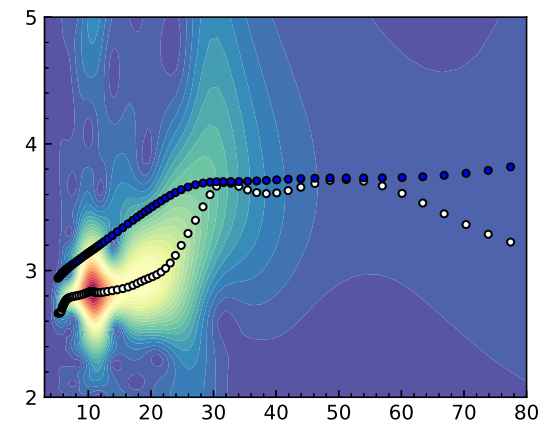

(b) ${ }^{e l l} \mathcal{I}_{3}^{D W}$

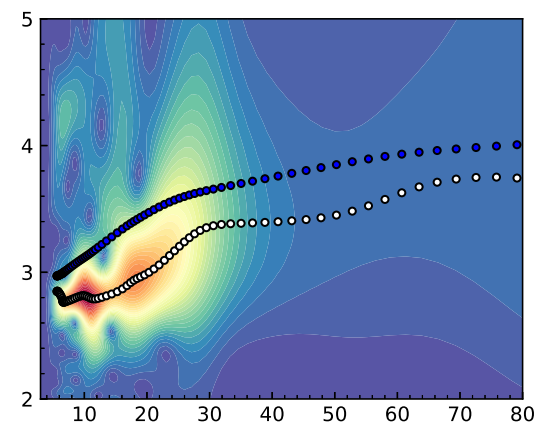

(d) $\mathcal{I}_{3}^{C W}$

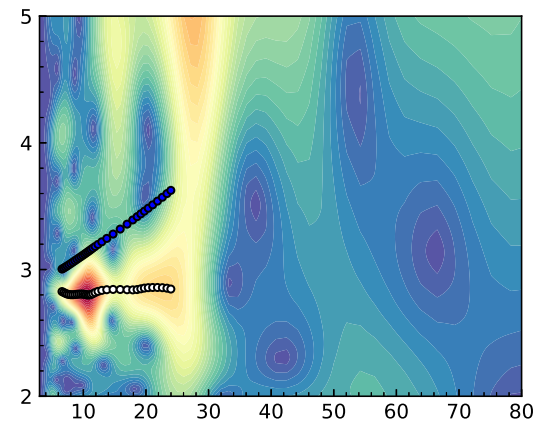

Period (s)

Figure 8. Frequency-time analysis (FTAN) diagrams for the receiver pair M07A-M15A using the waveforms from Fig. 6: (a) $\mathcal{I}_{2}^{A N}$, (b) $\mathcal{I}_{3}^{C W}$, (c) ${ }^{h y p} \mathcal{I}_{3}^{D W}$, and (d) ${ }^{\text {ell }} \mathcal{I}_{3}^{D W}$. White and blue circles are group and phase speed measurements, respectively. 

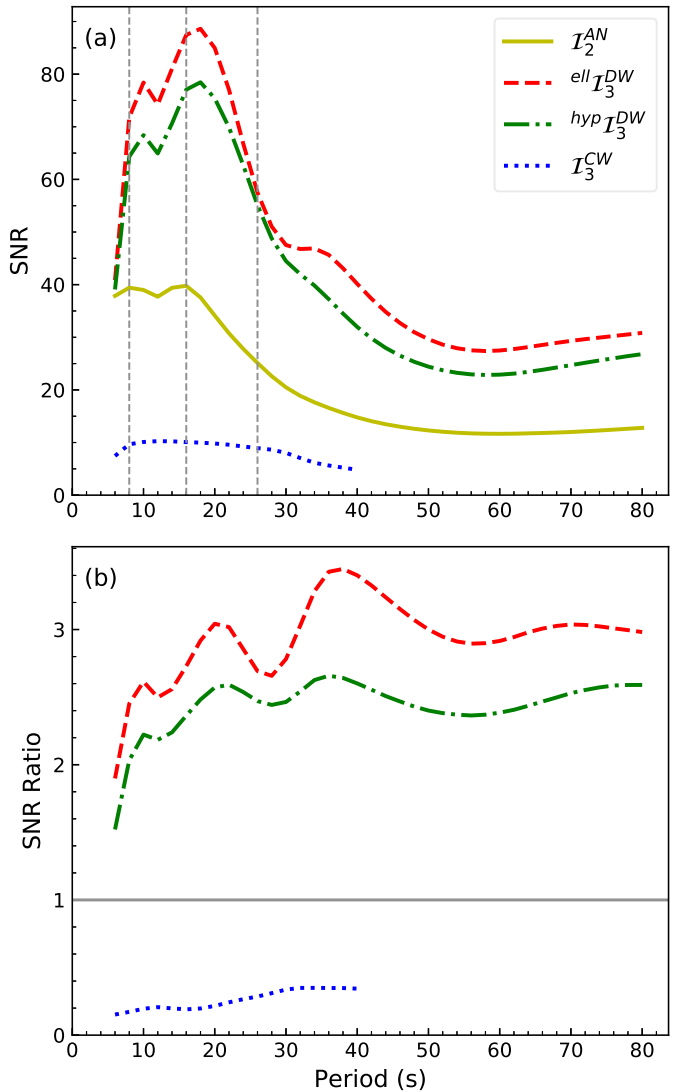

Figure 9. Signal-to-noise ratio (SNR) of estimated Green's functions for the different interferometric methods (see legend) plotted versus period. (a) Median of the SNR for each method taken over all measurements at each period. SNR generally decreases with period for all methods, but the highest SNR is from the three-station direct-wave method with an elliptical stationary phase zone $\left({ }^{e l l} \mathcal{I}_{3}^{D W}\right)$ and the lowest is from the three-station coda-wave method $\left(\mathcal{I}_{3}^{C W}\right)$. (b) Paths common to two-station and three-station interferometry in (a) are selected such that the ratio of the median SNR for each three-station method to that for the two-station method is shown. The direct-wave methods increase SNR relative to $\mathcal{I}_{2}^{A N}$ by a factor ranging from about 1.5 to 3 which grows with period, whereas the coda-wave method reduces SNR by a factor of 3-5. 

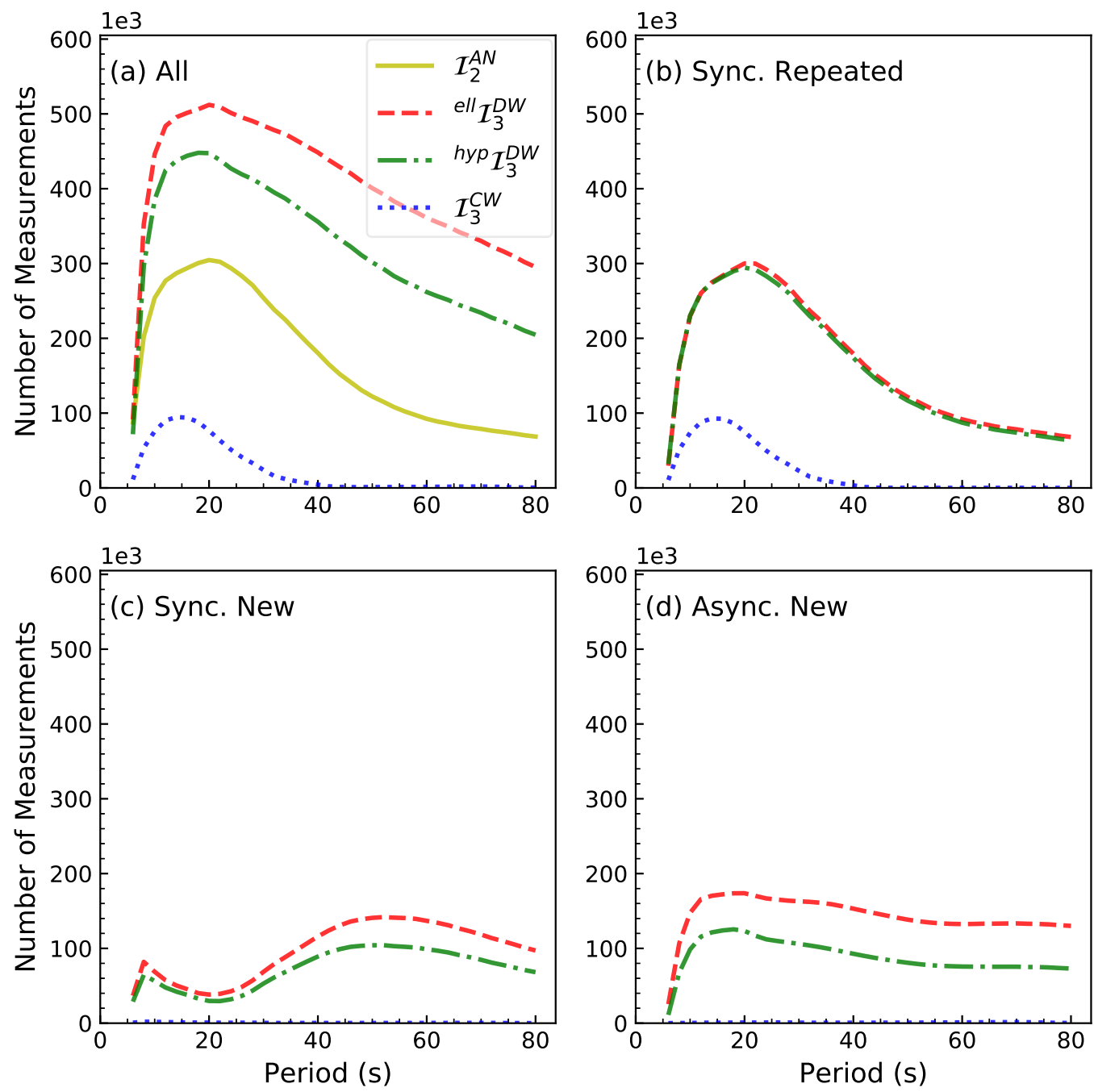

Figure 10. Number of resulting measurements (in thousands) versus period. (a) Number of accepted Rayleigh wave phase speed measurements plotted versus period for the different interferometric methods (see legend). The largest nunber of measurements is from the three-station direct-wave method with an elliptical stationary phase zone $\left({ }^{e l l} \mathcal{I}_{3}^{D W}\right)$ and the smallst number is from the three-station coda-wave method $\left(\mathcal{I}_{3}^{C W}\right)$. The total number of measurements can be broken into three parts, as shown in (b)-(d). (b) Number of measurements from $\mathcal{I}_{3}$ that exist for $\mathcal{I}_{2}^{A N}$. (c) Number of synchronous measurements from three-station interferometry methods $\left(\mathcal{I}_{3}\right)$ that are non-existent for two-station interferometry $\mathcal{I}_{2}^{A N}$ (because of low SNR). (d) Number of asynchronous measurements from $\mathcal{I}_{3}$ that are non-existent for $\mathcal{I}_{2}^{A N}$ (because of asynchrony). 

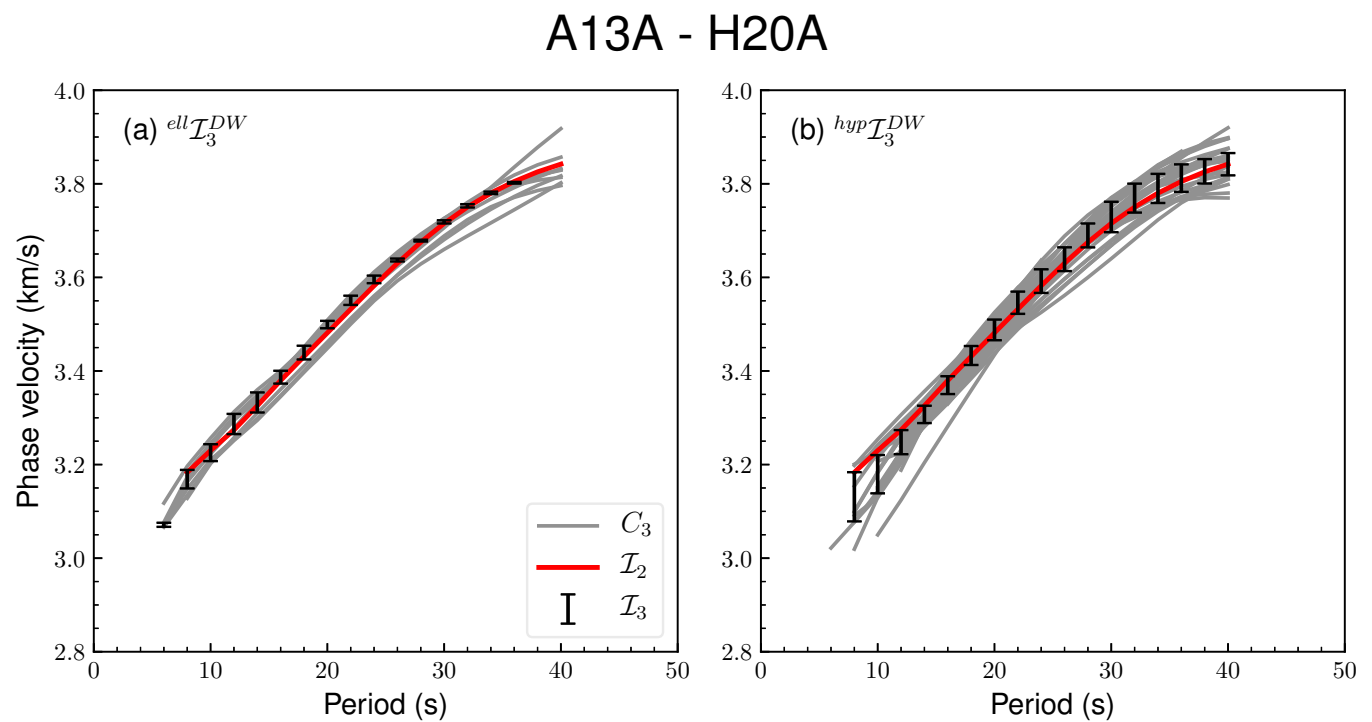

Figure 11. Examples of the de-biased Rayleigh wave phase speed curves for the receiverstation pair A13A (Polebridge, MT) and H20A (Greybull, WY) for the two three-station directwave methods: (a) ${ }^{e l l} \mathcal{I}_{3}^{D W}$ and (b) ${ }^{h y p} \mathcal{I}_{3}^{D W}$. Each gray curve is measured for a single sourcespecific interferogram $\left(C_{3}\right)$, where there are 9 source-stations for ${ }^{\text {ell }} \mathcal{I}_{3}^{D W}$ and 32 source-stations for ${ }^{h y p} \mathcal{I}_{3}^{D W}$. The mean and standard deviation of the constituent curves are plotted with the black error bars. The two-station ambient noise $\left(\mathcal{I}_{2}\right)$ dispersion curve is shown in red. 

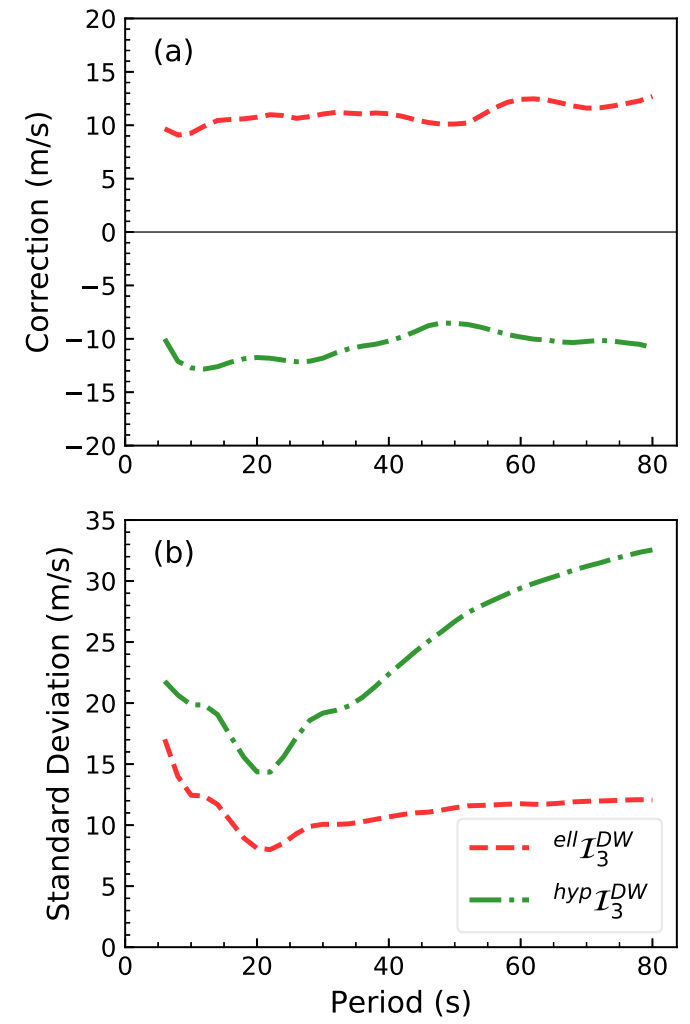

Figure 12. (a) Mean de-biasing correction averaged over all receiver-station pairs in the data set for ${ }^{e l l} \mathcal{I}_{3}^{D W}$ (red line) and ${ }^{h y p} \mathcal{I}_{3}^{D W}$ (green line). (b) Standard deviation of the de-biased dispersion curves averaged over all receiver-station pairs in the data set. 
Before correction
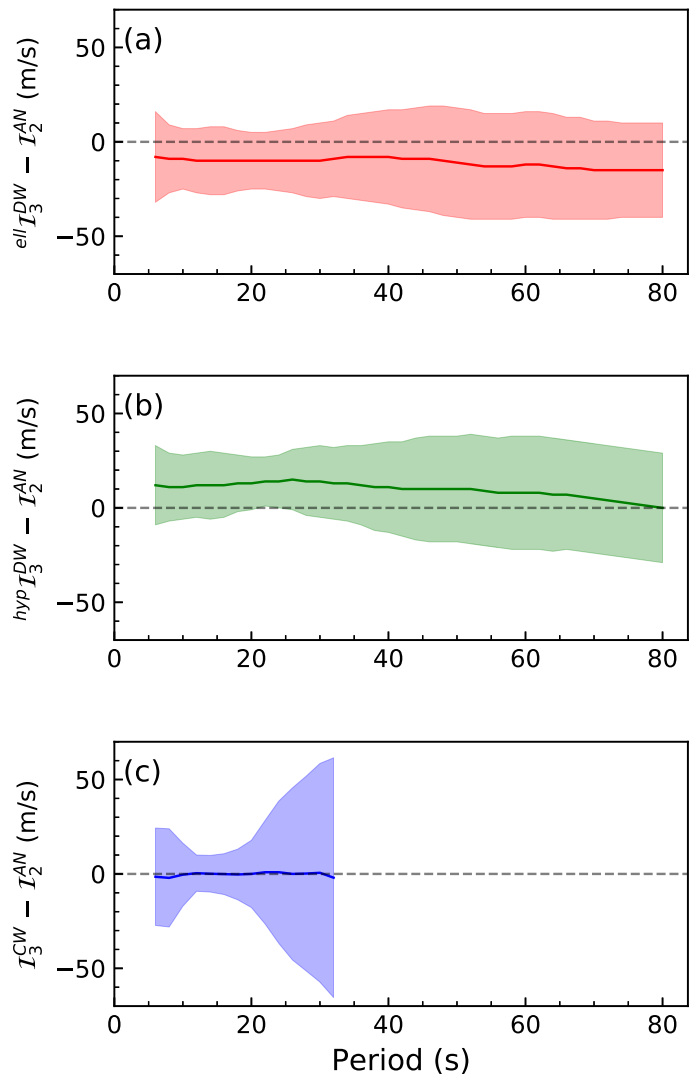

After correction
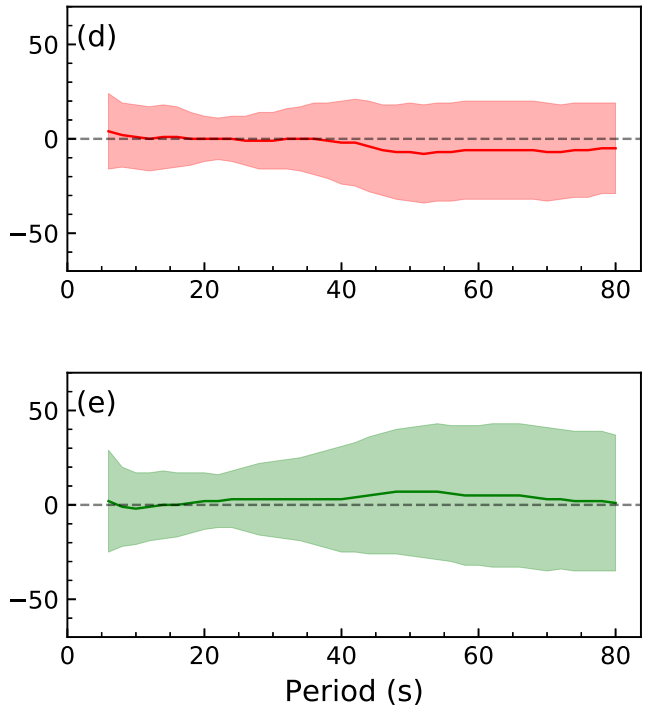

Figure 13. Mean and standard deviation of the difference between Rayleigh wave phase speed measurements from the three-station methods $\left(\mathcal{I}_{3}\right)$ and the two-station $\left(\mathcal{I}_{2}^{A N}\right)$ method. (a)-(c) No bias correction has been applied. Measurements from the direct-wave three-station methods $\left(\mathcal{I}_{3}^{D W}\right)$ are systematically shifted from the $\mathcal{I}_{2}^{A N}$ measurements, albeit with different signs, whereas the coda-wave measurements $\left(\mathcal{I}_{3}^{C W}\right)$ are not shifted relative to those from $\mathcal{I}_{2}^{A N}$. The standard deviation of the differences between the three-station and two-station measurements grow with period generally, but minimize around $20 \mathrm{~s}$. (d)-(e) Similar to (a)-(b), but the $\mathcal{I}_{3}^{D W}$ methods have been de-biased based on ray-theory. Systematic differences in Rayleigh wave phase speed measurements compared to the $\mathcal{I}_{2}^{A N}$ method are largely removed at periods below $40 \mathrm{~s}$, and are reduced at longer periods compared to the uncorrected values. The statistics are tabulated in

Tables 1, 2. 


\section{$10 \mathrm{~s}$}

(a)

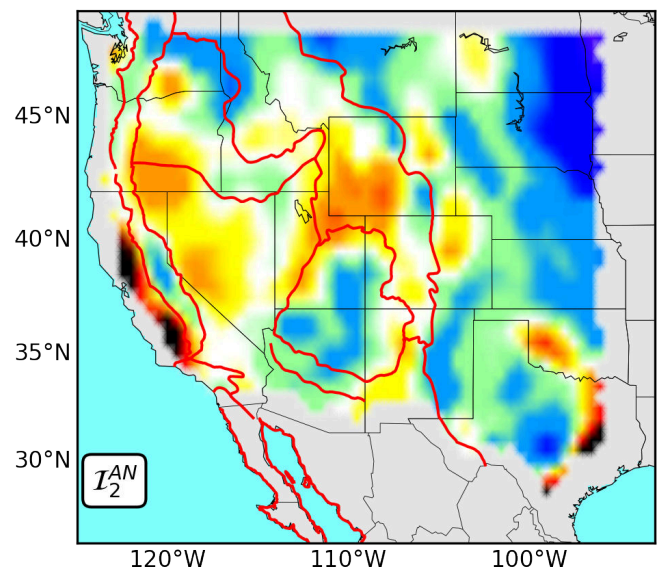

(c)

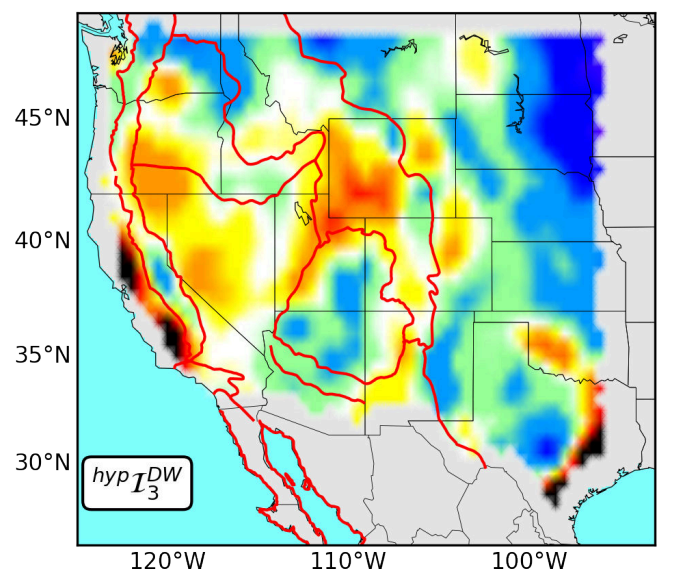

(b)

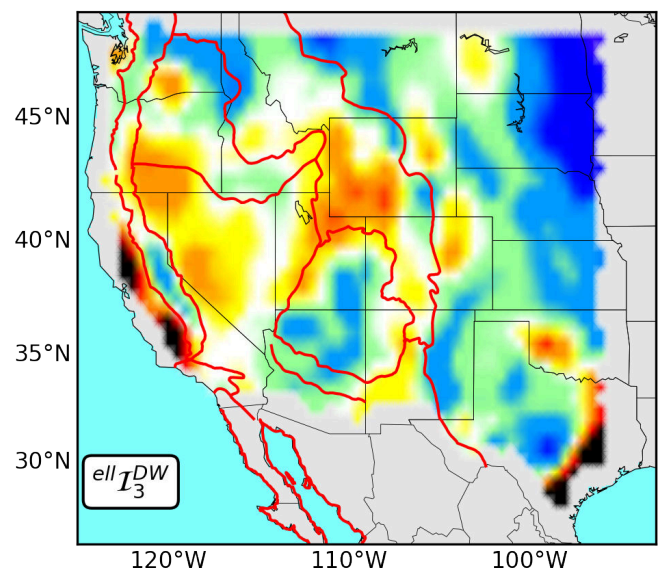

(d)

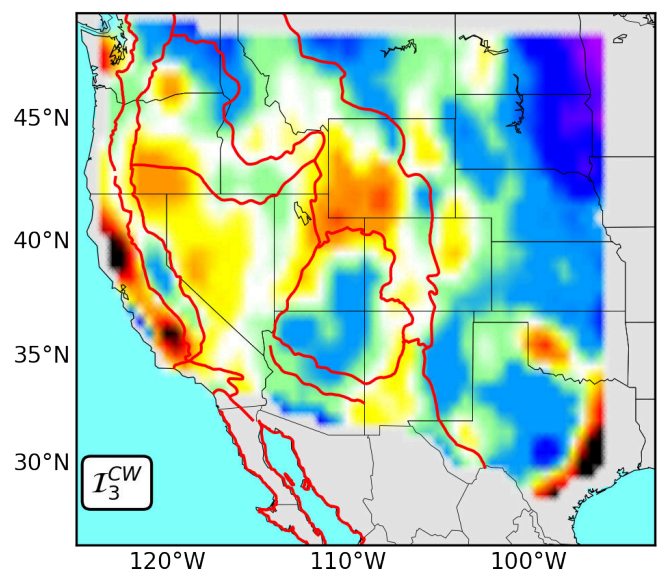

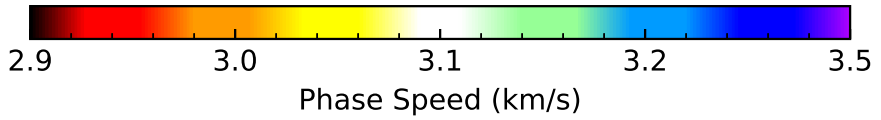

Figure 14. Rayleigh wave phase speed maps constructed with eikonal tomography at $10 \mathrm{~s}$ period using four different interferometric methods: (a) traditional two-station ambient noise interferometry $\left(\mathcal{I}_{2}^{A N}\right)$, (b) three-station direct-wave interferometry with elliptical stationary phase zone $\left({ }^{e l l} \mathcal{I}_{3}^{D W}\right)$, (c) three-station direct-wave interferometry with hyperbolic stationary phase zone $\left({ }^{h y p} \mathcal{I}_{3}^{D W}\right)$, and $(\mathrm{d})$ three-station coda-wave interferometry $\left(\mathcal{I}_{3}^{C W}\right)$. Red lines depict geological provinces (Fenneman \& Johnson, 1946). 
$20 \mathrm{~s}$

(a)

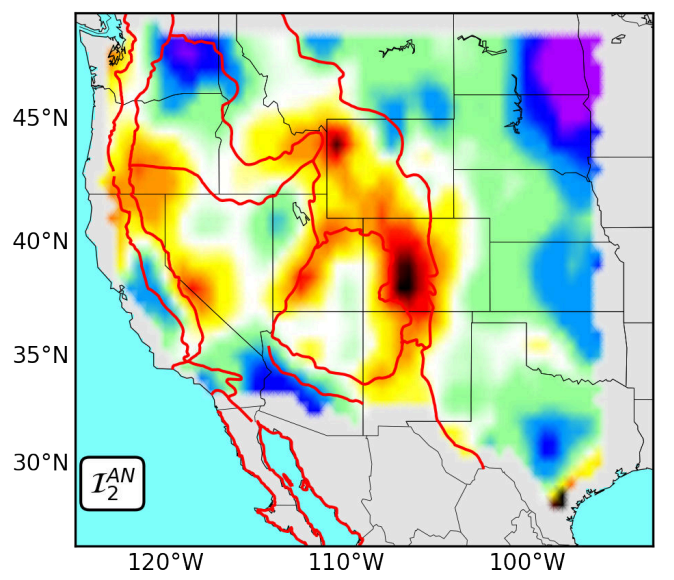

(c)

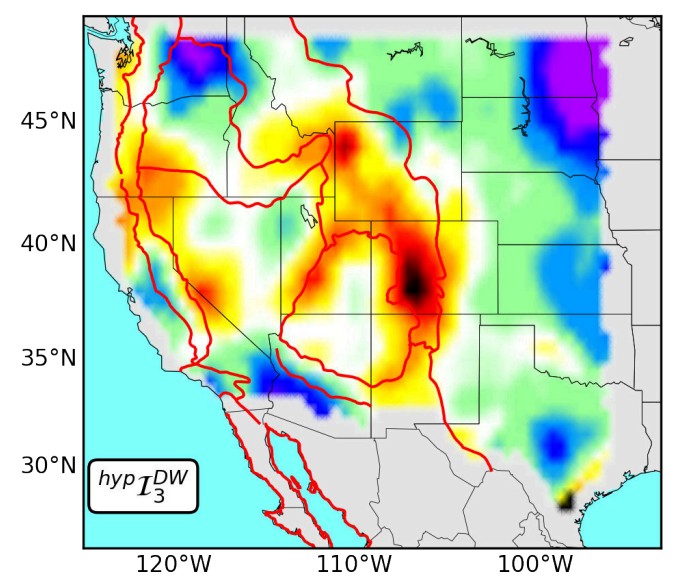

(b)

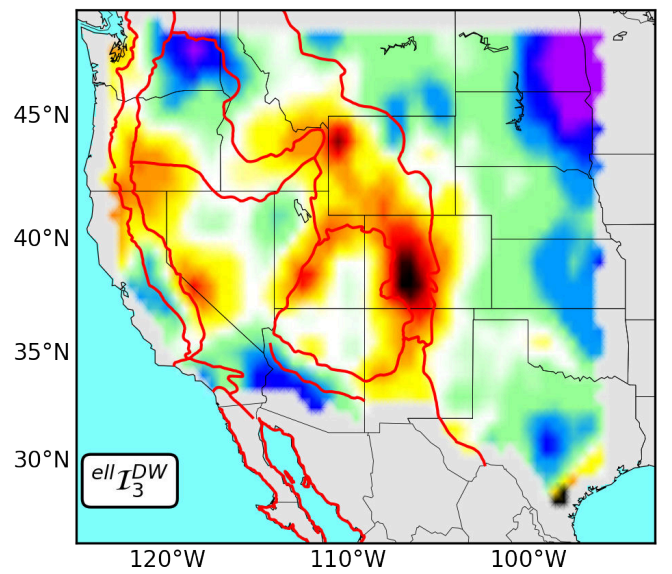

(d)

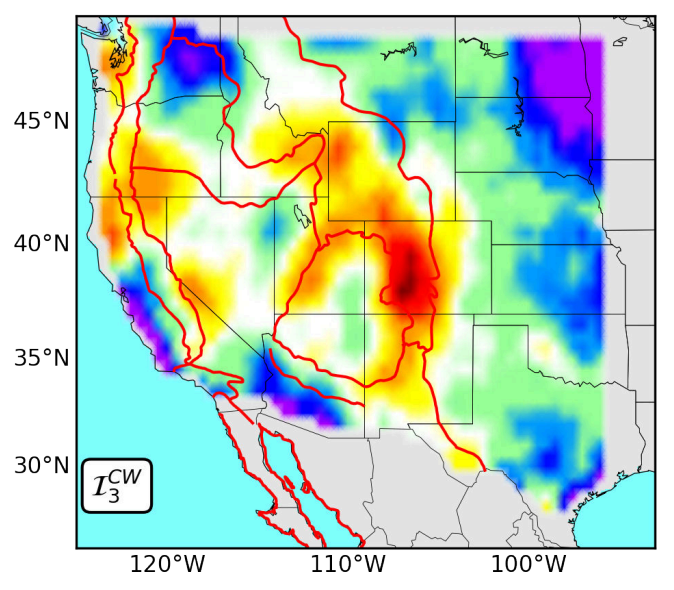

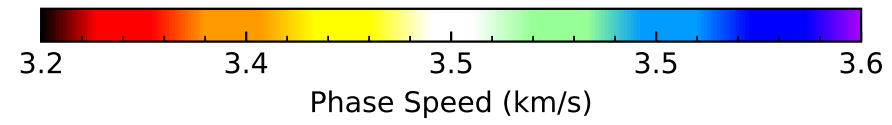

Figure 15. Similar to Fig. 14, but at 20 s period. 
$40 \mathrm{~s}$

(a)

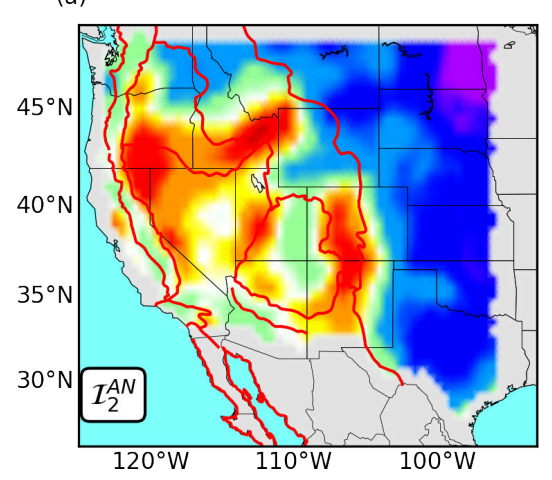

(b)

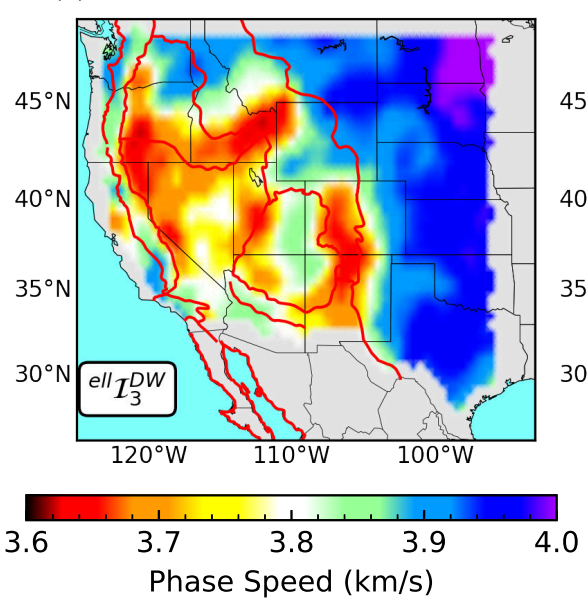

(c)

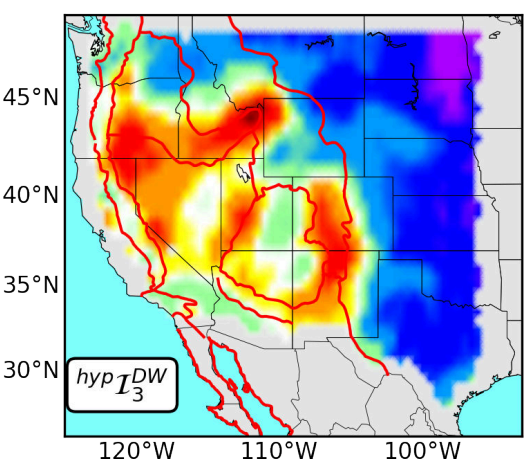

Figure 16. Similar to Fig. 14, but at a period of $40 \mathrm{~s} . \mathcal{I}_{3}^{C W}$ yielded too few measurements to produce a tomographic map.

\section{$60 \mathrm{~s}$}

(a)

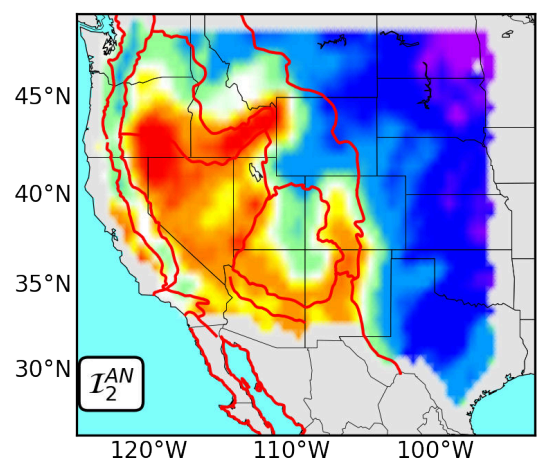

(b)

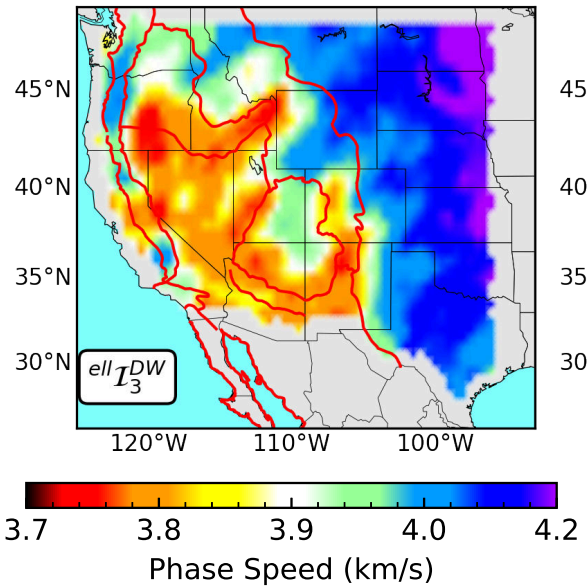

(c)

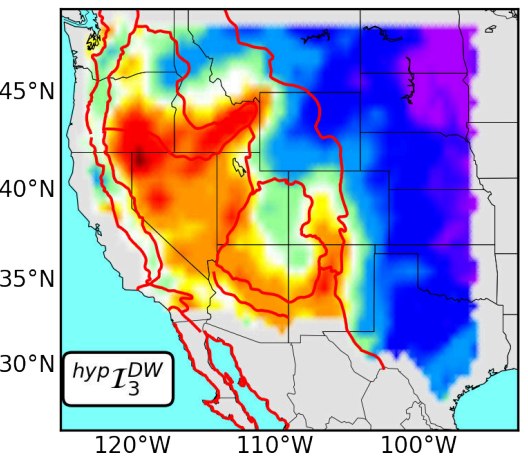

Figure 17. Similar to Fig. 16, but at a period of $60 \mathrm{~s}$. 


$$
\mathcal{I}_{3}^{C W}-\mathcal{I}_{2}^{A N}
$$

(a)

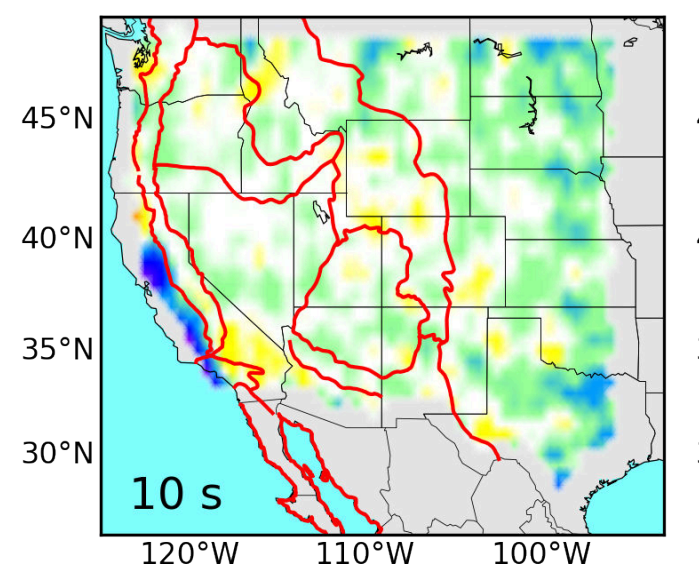

(b)

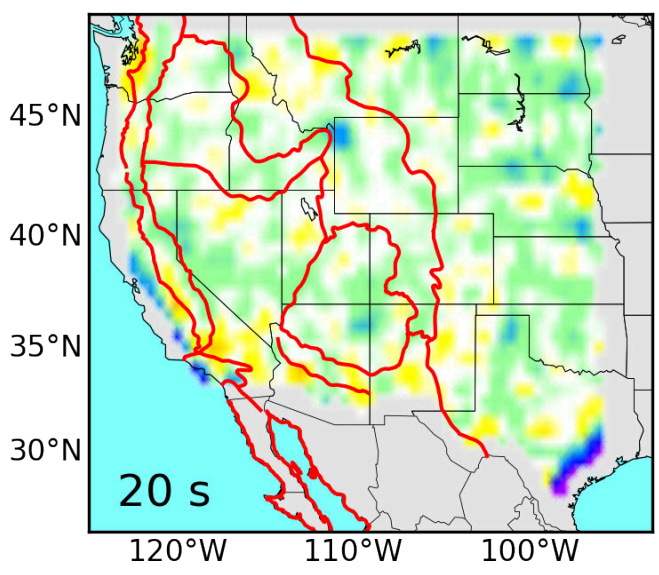

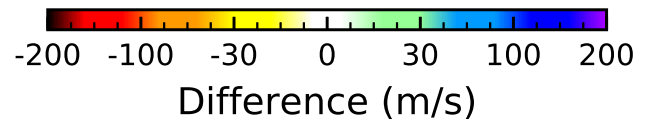

Figure 18. Differences in Rayleigh wave phase speed maps (Figs 14 and 15) between threestation coda-wave interferometry $\left(\mathcal{I}_{3}^{C W}\right)$ and two-station ambient noise interferometry $\left(\mathcal{I}_{2}^{A N}\right)$. $\mathcal{I}_{3}^{C W}$ yields too few measurements to produce tomographic maps at longer periods. 


$$
{ }^{\text {ell }} \mathcal{I}_{3}^{D W}-\mathcal{I}_{2}^{\text {AN }}
$$

(a)

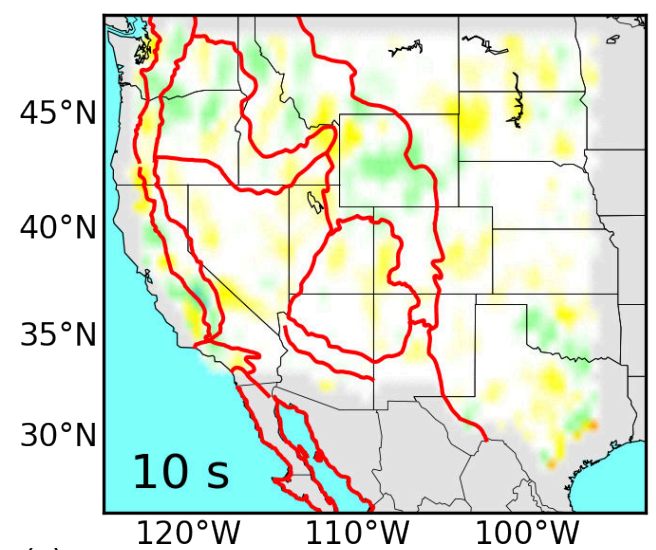

(c)

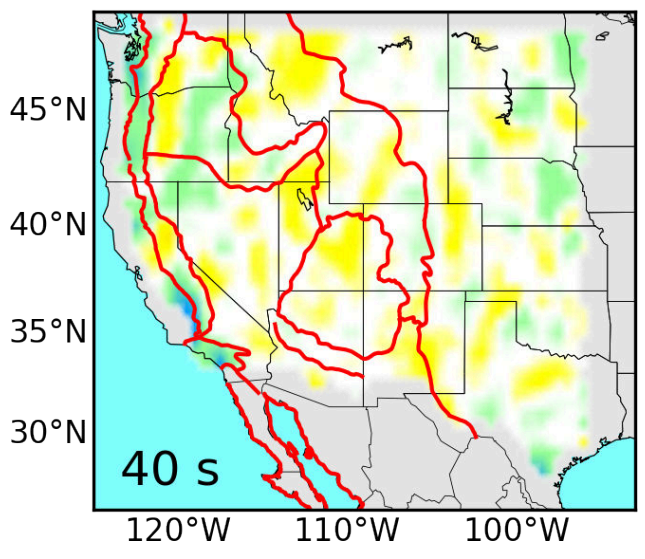

(b)

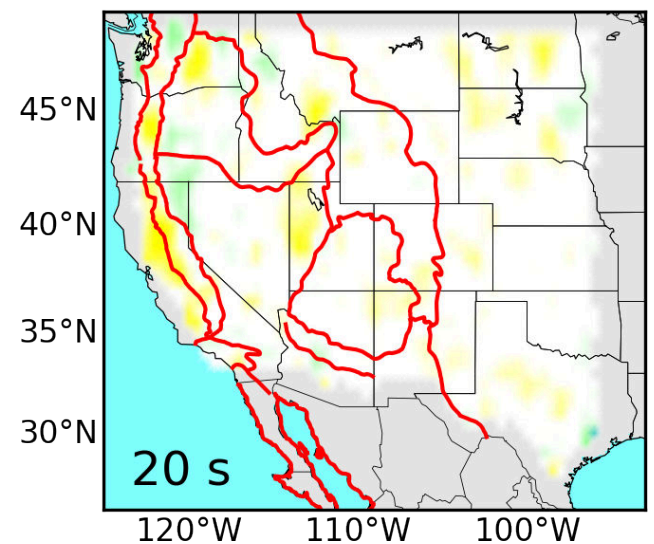

(d)

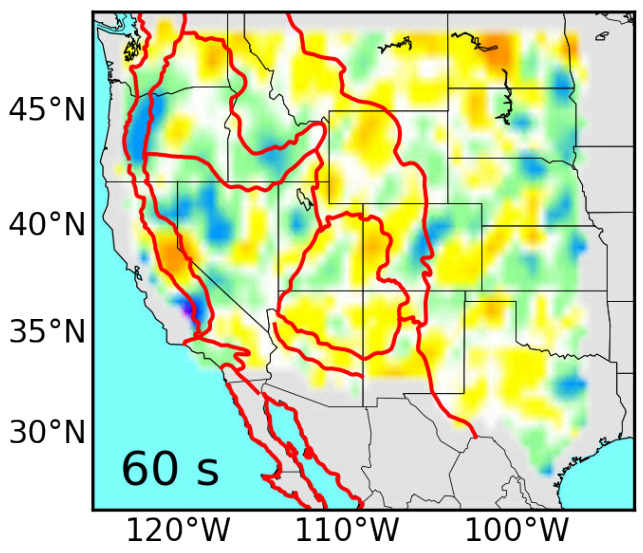

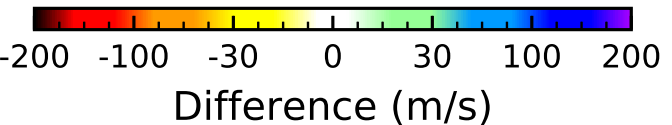

Figure 19. Similar to Fig. 18 except differences are between three-station direct-wave interferometry ${ }^{e l l} \mathcal{I}_{3}^{D W}$ and $\mathcal{I}_{2}^{A N}($ Figs 14 - 16), and results are presented at four periods: $10 \mathrm{~s}, 20 \mathrm{~s}$, $40 \mathrm{~s}$, and $60 \mathrm{~s}$. 


$$
{ }^{\text {hyp }} \mathcal{I}_{3}^{D W}-\mathcal{I}_{2}^{A N}
$$

(a)

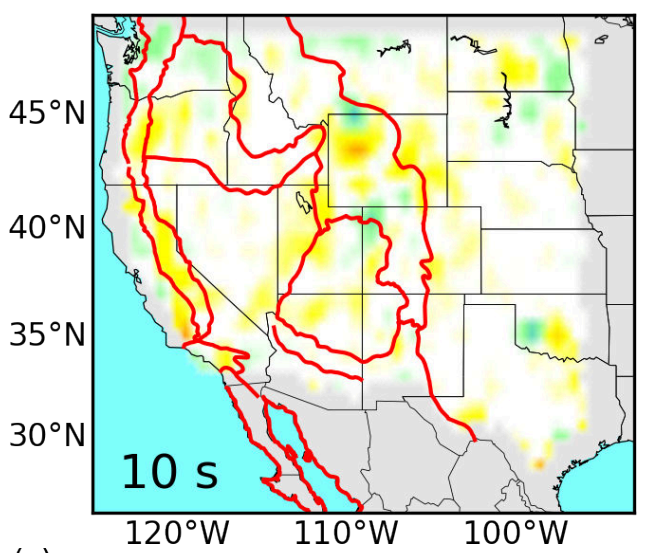

(c)

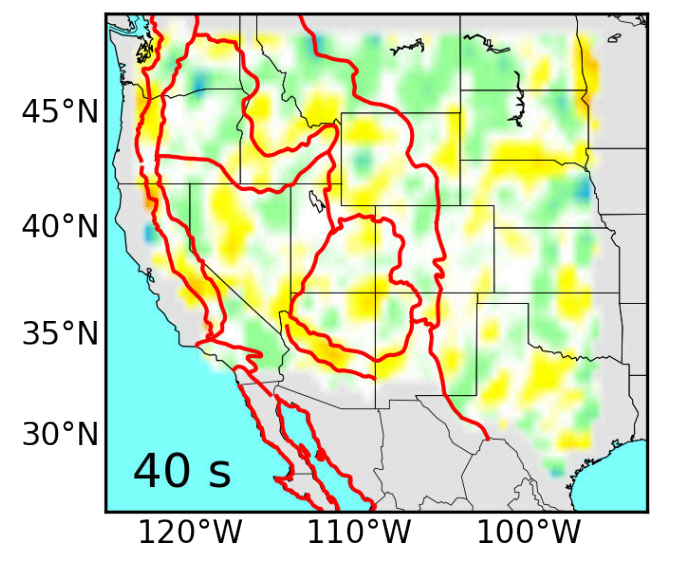

(b)

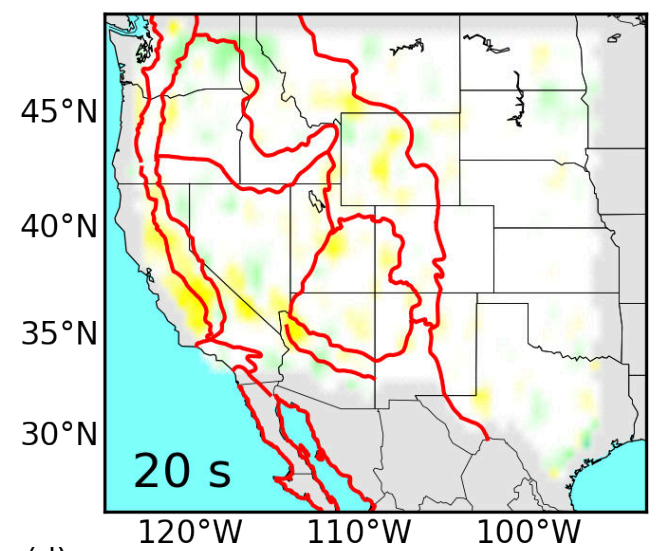

(d)

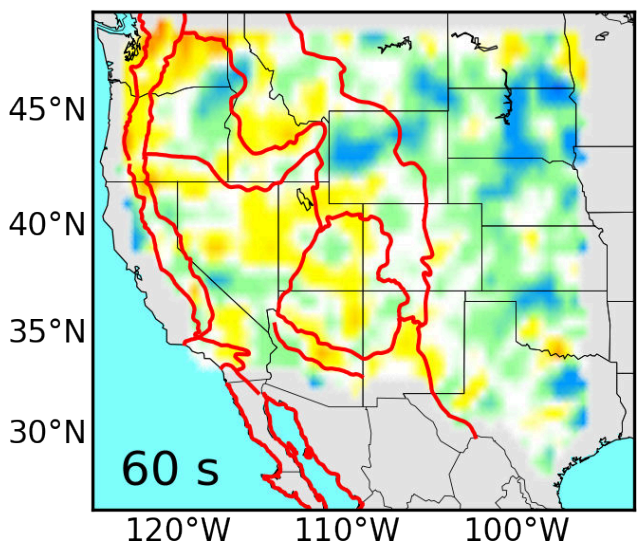

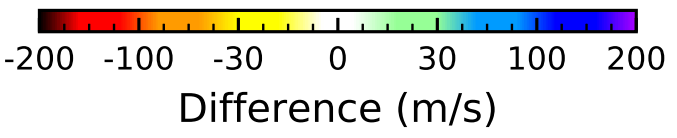

Figure 20. Similar to Fig. 19 except between ${ }^{h y p} \mathcal{I}_{3}^{D W}$ and $\mathcal{I}_{2}^{A N}$. 
(a) ${ }^{\text {ell }} \mathcal{I}_{3}^{D W}$

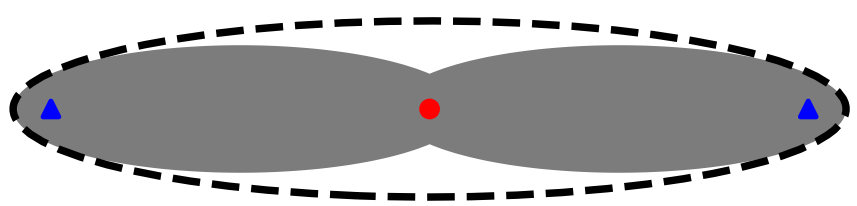

(b) ${ }^{\text {hyp }} \mathcal{I}_{3}^{D W}$

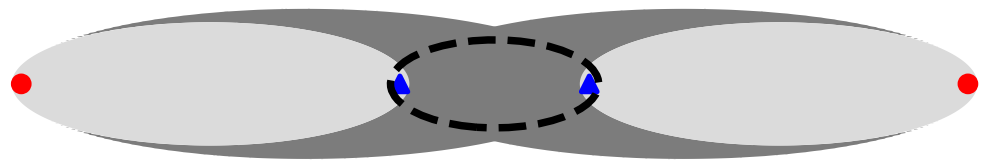

Figure 21. Schematic illustration contrasting the sensitivity kernels for ${ }^{\text {ell }} \mathcal{I}_{3}^{D W}$ and ${ }^{h y p} \mathcal{I}_{3}^{D W}$ with that for $\mathcal{I}_{2}$ which is shown as a Fresnel ellipse encompassing the two receiverstations (blue triangles) and is depicted with the dashed lines. (a) The sensitivity kernel for ${ }^{e l l} \mathcal{I}_{3}^{D W}$ is a superposition of the two elliptical Fresnel zones where the source-station (red dot) is at one focus and each of the receiver-stations are at the other foci. The resulting sensitivity kernel for ${ }^{e l l} \mathcal{I}_{3}^{D W}$ (grey region) is smaller than the kernel for $\mathcal{I}_{2}$ (zone encompassed by the dashed line). (b) The sensitivity kernel for ${ }^{h y p} \mathcal{I}_{3}^{D W}$ is the difference of the two elliptical Fresnel zones where each source-station (red dots) is at one focus and each of the receiver-stations is at the other focus. The resulting sensitivity kernel for ${ }^{h y p} \mathcal{I}_{3}^{D W}$ (grey region) is more complicated and larger than the kernel for $\mathcal{I}_{2}$ (zone encompassed by the dashed line). 

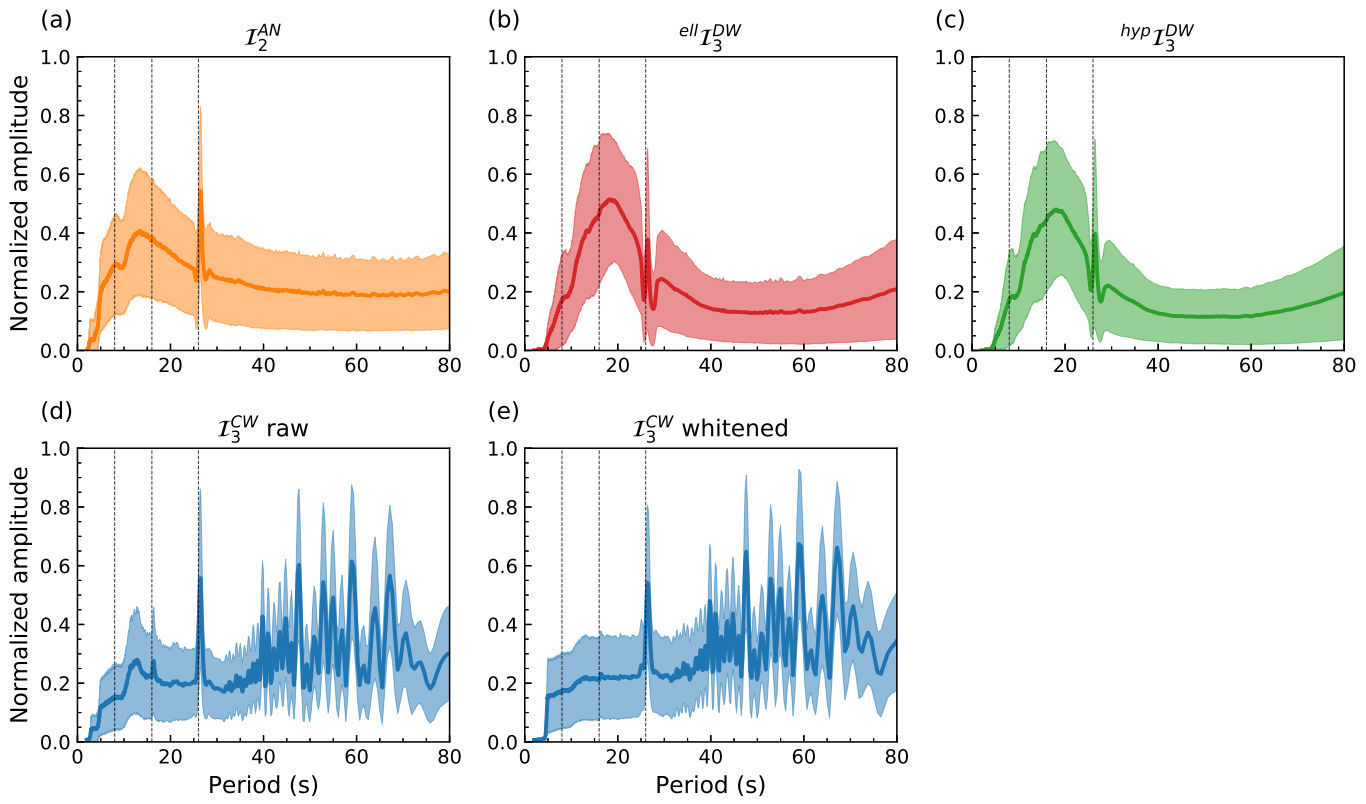

Figure 22. Mean (lines) and standard deviation (shaded areas) of spectra for (a) traditional two-station ambient noise interferometry $\left(\mathcal{I}_{2}^{A N}\right)$, (b) three-station direct-wave interferometry with elliptical stationary phase zone $\left({ }^{e l l} \mathcal{I}_{3}^{D W}\right)$, (c) three-station direct-wave interferometry with hyperbolic stationary phase zone $\left({ }^{h y p} \mathcal{I}_{3}^{D W}\right),(\mathrm{d})$ three-station coda-wave interferometry $\left(\mathcal{I}_{3}^{C W}\right)$ without spectral whitening, and (e) $\mathcal{I}_{3}^{C W}$ with spectral whitening. Dashed lines mark the secondary microseism peak ( $8 \mathrm{~s})$, the primary microseism peak (16 s), and the $26 \mathrm{~s}$ microseism. The $26 \mathrm{~s}$ microseism shows a peak across all methods. Whitening of $\mathcal{I}_{3}^{C W}$ only makes the spectra flatter at short periods but does not eliminate the 26 s peak. The spectra of $\mathcal{I}_{3}^{C W}$ show strong variability at long periods which is not observed in other methods. Whitening of $\mathcal{I}_{3}^{C W}$ does not reduce the variability at long periods. 